\title{
Application of the One-Way Navier-Stokes (OWNS) Equations to Hypersonic Boundary Layers
}

\author{
Omar Kamal* \\ California Institute of Technology, Pasadena, California, 91106, United States \\ Georgios Rigas ${ }^{\dagger}$ \\ Imperial College London, South Kensington, London, SW7 2AZ, United Kingdom \\ Matthew T. Lakebrink \\ The Boeing Company, Hazelwood, MO, 63042, United States \\ Tim Colonius $\S$ \\ California Institute of Technology, Pasadena, California, 91106, United States
}

\begin{abstract}
Prediction of linear amplification of disturbances in hypersonic boundary layers is challenging due to the presence and interactions of discrete modes (e.g. Tollmien-Schlichting and Mack) and continuous modes (entropic, vortical, and acoustic). While DNS and global analysis can be used, the large grids required make the computation of optimal transient and forced responses expensive, particularly when a large parameter space is required. At the same time, parabolized stability equations are non-convergent and unreliable for problems involving multi-modal and non-modal interactions. In this work, we apply the One-Way Navier-Stokes (OWNS) equations to hypersonic boundary layers. OWNS is based on a rigorous, approximate parabolization of the equations of motion that removes disturbances with upstream group velocity using a high-order recursive filter. We extend the original algorithm by considering non-orthogonal body-fitted curvilinear coordinates and incorporate full compressibility with temperature-dependent fluid properties. We validate the results by comparing to DNS data for a flat plate and sharp cone, and to LST results for local disturbances on the centerline of the HIFiRE-5 elliptic cone. OWNS provides DNS-quality results for the former flows at a small fraction of the computational expense.
\end{abstract}

\section{Nomenclature}

$(\xi, \eta, \zeta)=$ Body-fitted streamwise, wall-normal, and spanwise coordinates

$(x, y, z)=$ Global Cartesian coordinates

$N_{\xi} \quad=\quad$ Number of points along the streamwise coordinate

$N_{\eta} \quad=\quad$ Number of points along the wall-normal coordinate

$N_{\zeta} \quad=\quad$ Number of points along the spanwise coordinate

$M_{\infty} / M_{e}=$ Freestream/boundary-layer edge Mach number

$T_{\infty} / T_{e} \quad=$ Freestream/boundary-layer edge temperature

$R e_{\infty} / R e_{e}=$ Freestream/boundary-layer edge unit Reynolds number defined as $\frac{\rho_{\infty} u_{\infty}}{\mu_{\infty}} / \frac{\rho_{e} u_{e}}{\mu_{e}}$

$p_{\infty} / p_{e}=$ Freestream/boundary-layer edge pressure

$a_{\infty} / a_{e} \quad=$ Freestream/boundary-layer edge speed of sound

$\rho_{\infty} / \rho_{e} \quad=$ Freestream/boundary-layer edge density

$k_{\infty} / k_{e}=$ Freestream/boundary-layer edge thermal conductivity

$\mu_{\infty} / \mu_{e} \quad=$ Freestream/boundary-layer edge dynamic viscosity

$c_{p_{\infty}} / c_{p_{e}}=$ Freestream/boundary-layer edge specific heat ratio at constant pressure

\footnotetext{
*PhD Candidate, Mechanical and Civil Engineering, AIAA Student Member.

${ }^{\dagger}$ Lecturer, Department of Aeronautics, AIAA Member.

$¥$ Aerodynamics Engineer, AIAA Senior Member.

${ }^{\S}$ Frank and Ora Lee Marble Professor of Mechanical Engineering, Mechanical and Civil Engineering, Associate Fellow AIAA.
} 


$\begin{array}{ll}\mathbf{u} & =\text { Velocity vector defined as }\left[u_{\xi}, u_{\eta}, u_{\zeta}\right]^{T} \\ \alpha & =\text { Complex streamwise wavenumber defined as } \alpha_{r}+i \alpha_{i} \\ \beta & =\text { Spanwise wavenumber } \\ N_{b} & =\text { Order of one-way approximation for OWNS } \\ T_{w} & =\text { Wall temperature } \\ v & =\text { Specific volume defined as } \frac{1}{\rho} \\ \kappa & =\text { Bulk viscosity } \\ \gamma & =\text { Specific heat ratio } \\ R & =\text { Gas constant } \\ \delta_{0} & =\text { Inlet Blasius length scale defined as } \sqrt{\frac{\mu_{\infty} \xi_{0}}{\rho_{\infty} u_{\infty}}} \\ f & =\text { Disturbance frequency } \\ \omega & =\text { Angular disturbance frequency } \\ F & =\text { Non-dimensional frequency defined as } \frac{\omega \mu_{\infty}}{\rho_{\infty} u_{\infty}^{2}} \\ c & =\text { Complex phase speed defined as } \frac{\omega}{\alpha}=c_{r}+i c_{i} \\ N & =\text { Disturbance amplification factor }\end{array}$

\section{Introduction}

Developing an in-depth understanding of hypersonic laminar-to-turbulent transition will help formulate methods to control aerodynamic and thermal loads. For example, due to the difficulty of predicting the onset of turbulence, the take-off mass of the US National Aerospace Plane (NASP/X-30) was affected by a factor of two or more [1]. Thus, it is of utmost importance to understand the state of the aerodynamic boundary layer throughout the flight trajectory for optimal vehicular design. We will first review the fundamentals of high-speed boundary-layer transition and thereafter describe the current tools used to predict the onset of transition.

For idealized flow conditions, i.e. slender geometries experiencing minor pressure gradients (if at all) and low freestream perturbations, at subsonic and low supersonic speeds, natural transition is due to the first mode which is characterized by the amplification of Tollmien-Schlichting (T-S) waves [2]. This occurs when the boundary layer is receptive to infinitesimal disturbances (e.g. surface roughness). The disturbance waves, which are intrinsically hydrodynamic, amplify as they travel downstream until they reach a finite amplitude of $\approx 1 \%$ of the freestream velocity in which then three-dimensional velocity and pressure fluctuations appear along with hairpin eddies [1, 3]. The flow then exhibits nonlinear breakdown where the streamwise stretched vortices cascade energy to smaller structures. Turbulent spots begin to appear after which the flow becomes fully turbulent.

In contrast, at hypersonic speeds $(\mathrm{M}>4)$, natural transition is far more complex due to the presence and interaction of multiple discrete modes including the first, second, and higher-order modes as denoted by Mack [4, 5]. Second and higher-order modes are characterized by convectively-amplified streamwise-propagating acoustic disturbances usually in the ultrasonic range. Several numerical [6-8] and experimental [9. 16] works have substantiated the existence of such instabilities. These disturbances approximately behave as acoustic waves reflecting between the solid wall and the relative sonic line [2]. The second Mack mode is usually the dominant instability in zero-pressure-gradient boundary layers at hypersonic speeds with insulated walls. Even with the aforementioned simplified conditions, the second mode becomes unstable due to a complex synchronization of fast (Mode F) and slow (Mode S) discrete modes that originate from upstream/downstream acoustic branches relative to a supersonic local velocity [17]. Additionally, it has been shown that the first mode along with other instabilities, such as the concave-wall Görtler instability [18], the supersonic mode [19], and three-dimensional crossflow instability [20, 21] interact with and/or modify the acoustic properties of the second mode (e.g. Görtler-modified azimuthal Mack-modes [22]). Thus, efficient predictive tools capable of capturing these multi-modal instabilities are critical for engineering design.

Cool surfaces can destabilize the second Mack mode at even lower Mach numbers. Bitter and Shepherd [23] demonstrated that Mode F (the fast acoustic mode) can become destabilized over a broad range of frequencies. Under high wall-cooling, this mode can synchronize with the slow acoustic branch causing an acoustic emission at a Mach angle corresponding to the relative phase speed. This additional mode, referred to as the supersonic mode [19], can elongate and modulate the disturbance within the boundary layer [24]. Thus, high-speed transition with the presence of wall-cooling adds an additional layer of complexity with the potential involvement of the supersonic mode.

Current tools to predict boundary-layer transition include direct numerical simulations (DNS) and its linear counterparts such as global stability analysis, linear stability theory (LST), and parabolized stability equations (PSE). 
Although global methods are most accurate, they are computationally intensive, especially with complex geometries. LST is more computationally efficient but typically employs the parallel-flow assumption that introduces error as seen in [25], which studies the stability characteristics of a Mach 4.5 flat-plate boundary layer. PSE may seem to be the optimal method between DNS and LST, but there are intrinsic disadvantages. Instead of formally deriving a one-way operator, PSE achieves a stable spatial march by numerically damping all upstream-propagating waves, as well as a subset of the downstream-propagating ones, by using an implicit streamwise discretization along with a restriction on the minimum step-size, or by explicitly adding damping terms to the equations [26]. In either form, the associated damping prevents the upstream waves from destabilizing the spatial march, but also has the unintended consequence of damping and distorting, to differing degrees, all of the downstream waves. This has, in turn, profound negative consequence for non-modal instabilities that are associated with an interacting group of stable modes and for flows with more rapid streamwise evolution [26]. In essence, using PSE and LST as engineering tools for boundary-layer transition prediction is suboptimal due to their inability to accurately model receptivity, non-modal and multi-modal instabilities along with the synchronization and coalescence of discrete modes with continuous branches which is relevant for hypersonic boundary layers [23, 24].

We alleviate the aforementioned deficiencies in PSE by introducing a novel marching technique, the One-Way Navier-Stokes (OWNS) equations. Originally developed for hyperbolic equations (e.g. Euler), OWNS employs a rigorous parabolization technique to generate well-posed, one-way approximations [27]. Efficient (fast) approximations of the resulting operator can then be made using recursive filters that were originally developed for non-reflecting boundary conditions. Aside from the parabolization itself, and unlike PSE, the numerics are convergent and not restricted to a dominant wavelength. OWNS was extended to wall-bounded flows and validated by comparison with PSE and DNS for a variety of two- and three-dimensional flows [28].

In the present paper, we further extend the OWNS technique to implement full compressibility, temperature-dependent fluid properties, and a general non-orthogonal curvilinear coordinate system that allows it to be applied to more complex geometries. We validate the methodology by studying linear amplification of disturbances in hypersonic boundary layers and compare results to LST, PSE, and DNS. We consider a flat plate, a sharp cone, and local analysis along the centerline of the HIFiRE-5 elliptic cone.

\section{Methodology}

\section{A. Linearized equations of motion}

We begin with the compressible Navier-Stokes equations for an ideal gas

$$
\begin{aligned}
& \frac{D v}{D t}-v(\nabla \cdot \mathbf{u})=0, \\
& \frac{D \mathbf{u}}{D t}+v \nabla p=\frac{v}{R e} \nabla \cdot(2 \mu S+\kappa(\nabla \cdot \mathbf{u}) I), \\
& \frac{D p}{D t}+\gamma p(\nabla \cdot \mathbf{u})=\frac{\gamma-1}{R \operatorname{PrRe}} \nabla \cdot(k \nabla p v)+\frac{\gamma-1}{\operatorname{Re}}\left(\kappa(\nabla \cdot \mathbf{u})^{2}+2 \mu S: \nabla \mathbf{u}\right), \\
& S=\frac{1}{2}\left(\nabla \mathbf{u}+(\nabla \mathbf{u})^{T}-\frac{2}{3}(\nabla \cdot \mathbf{u}) I\right), \\
& p=R T / v \\
& \operatorname{Re}=\frac{\rho_{\infty}^{*} a_{\infty}^{*} \delta_{0}^{*}}{\mu_{\infty}^{*}}, \\
& \operatorname{Pr}=\frac{\mu_{\infty}^{*} c_{p_{\infty}}^{*}}{k_{\infty}^{*}},
\end{aligned}
$$

where the variables have been non-dimensionalized by $\delta_{0}^{*}, a_{\infty}^{*}, \rho_{\infty}^{*}, k_{\infty}^{*}, c_{p_{\infty}}^{*}$, and $\mu_{\infty}^{*}$. We follow the two-step transformation from the Cartesian coordinate system to the physical non-orthogonal curvilinear coordinate system outlined in [29] and linearize all equations about a time-independent base flow such that

$$
q(\xi, \eta, \zeta, t)=\bar{q}(\xi, \eta, \zeta)+q^{\prime}(\xi, \eta, \zeta, t)
$$


where $q=\left[v, u_{\xi}, u_{\eta}, u_{\zeta}, p\right]^{T}$ is the state vector. Due to algebraic complexity, we have automated this transformation using the Mathematica software and present the general matrix form of the resulting linear equations

$$
\begin{gathered}
\frac{\partial q^{\prime}}{\partial t}+A_{\xi, i v s} \frac{\partial q^{\prime}}{\partial \xi}+A_{\eta, i v s} \frac{\partial q^{\prime}}{\partial \eta}+A_{\zeta, i v s} \frac{\partial q^{\prime}}{\partial \zeta}+A_{i v s} q^{\prime}=A_{\xi, v i s} \frac{\partial q^{\prime}}{\partial \xi}+A_{\eta, v i s} \frac{\partial q^{\prime}}{\partial \eta}+A_{\zeta, v i s} \frac{\partial q^{\prime}}{\partial \zeta}+ \\
A_{v i s} q^{\prime}+B_{\xi \xi} \frac{\partial^{2} q^{\prime}}{\partial \xi^{2}}+B_{\eta \eta} \frac{\partial^{2} q^{\prime}}{\partial \eta^{2}}+B_{\zeta \zeta} \frac{\partial^{2} q^{\prime}}{\partial \zeta^{2}}+B_{\xi \eta} \frac{\partial^{2} q^{\prime}}{\partial \xi \partial \eta}+B_{\xi \zeta} \frac{\partial^{2} q^{\prime}}{\partial \xi \partial \zeta}+B_{\eta \zeta} \frac{\partial^{2} q^{\prime}}{\partial \eta \partial \zeta} .
\end{gathered}
$$

Note that all of the aforementioned base flow coefficient matrices are functions of $(\xi, \eta, \zeta)$. After discretizing in the $\eta$ and possibly $\zeta$ directions (depending on the problem type) using a summation-by-parts fourth-order central finite-difference scheme [30], we have

$$
\frac{d q^{\prime}}{d t}+A_{\xi, i v s} \frac{d q^{\prime}}{d \xi}+B q^{\prime}=C \frac{d q^{\prime}}{d \xi}+B_{\xi \xi} \frac{d^{2} q^{\prime}}{d \xi^{2}},
$$

where the discrete operators $B[\xi]$ and $C[\xi]$ are defined as

$$
\begin{gathered}
B[\xi]=A_{\eta, i v s} \frac{\partial}{\partial \eta}+A_{\zeta, i v s} \frac{\partial}{\partial \zeta}+A_{i v s}-\left(A_{\eta, v i s} \frac{\partial}{\partial \eta}+A_{\zeta, v i s} \frac{\partial}{\partial \zeta}+A_{v i s}+B_{\eta \eta} \frac{\partial^{2}}{\partial \eta^{2}}+B_{\zeta \zeta} \frac{\partial^{2}}{\partial \zeta^{2}}+B_{\eta \zeta} \frac{\partial^{2}}{\partial \eta \partial \zeta}\right), \\
C[\xi]=A_{\xi, v i s}+B_{\xi, \eta} \frac{\partial}{\partial \eta}+B_{\xi, \zeta} \frac{\partial}{\partial \zeta} .
\end{gathered}
$$

We now transform the discretized equation to characteristic space via the transformation

$$
\phi(\xi, \eta, \zeta, t)=T(\xi, \eta, \zeta) q^{\prime}(\xi, \eta, \zeta, t), \quad \widetilde{A}_{\xi, i v s}=T A_{\xi, i v s} T^{-1},
$$

where the columns of $T$ are the eigenvectors of $A_{\xi, i v s}$. The discretized characteristic equation reads

$$
\frac{d \phi}{d t}+\widetilde{A}_{\xi, i v s} \frac{d \phi}{d \xi}+\widetilde{B} \phi=\widetilde{C} \frac{d \phi}{d \xi}+\widetilde{B}_{\xi \xi} \frac{d^{2} \phi}{d \xi^{2}}+\widetilde{D} \phi
$$

where

$$
\begin{gathered}
\widetilde{B}=T B T^{-1}+T A_{\xi, i v s} \frac{d T^{-1}}{d \xi}, \quad \widetilde{C}=T C T^{-1}+2 T B_{\xi \xi} \frac{d T^{-1}}{d \xi}, \\
\widetilde{B}_{\xi \xi}=T B_{\xi \xi} T^{-1}, \quad \widetilde{D}=T C \frac{d T^{-1}}{d \xi}+T B_{\xi \xi} \frac{d^{2} T^{-1}}{d \xi^{2}} .
\end{gathered}
$$

The RHS of Eq. 8 corresponds to streamwise viscous terms which will be discretized explicitly when performing the spatial march and thus we can denote it as a forcing term

$$
f_{\xi, v i s}=\widetilde{C} \frac{d \phi}{d \xi}+\widetilde{B}_{\xi \xi} \frac{d^{2} \phi}{d \xi^{2}}+\widetilde{D} \phi
$$

In preparation for the parabolization, we rewrite the equations in the frequency domain via the Laplace transform yielding

$$
s \widehat{\phi}+\widetilde{A}_{\xi, i v s} \frac{\mathrm{d} \widehat{\phi}}{\mathrm{d} \xi}+\widetilde{B} \widehat{\phi}=\widehat{f}_{\xi, v i s},
$$

and formally isolate the marching variable, $\xi$, by writing

$$
\frac{\mathrm{d} \widehat{\phi}}{\mathrm{d} \xi}=M \widehat{\phi}+\widehat{h}+\widehat{g},
$$

where 


$$
\begin{gathered}
s=-i \omega, \\
M(\xi, \omega)=-\widetilde{A}_{\xi, i v s}^{-1}(-i \omega I+\widetilde{B}), \\
\widehat{h}(\xi)=\widetilde{A}_{\xi, i v s}^{-1} \widehat{f}, \\
\widehat{g}(\xi)=\widetilde{A}_{\xi, i v s}^{-1} \widehat{f_{\xi}, v i s},
\end{gathered}
$$

and $\widehat{f}$ is an arbitrary volumetric forcing (in characteristic space) that can be additionally applied to excite the boundary layer. Eq. 12 is still exact, but cannot be solved as an initial value problem (IVP) in $\xi$ because $M$ has eigenvalues of both signs. In PSE, this equation is regularized to damp the upstream modes, whereas in OWNS, described in the next section, the equation is parabolized by filtering out the modes with upstream group velocity. Hereon, we will omit the symbol, but all perturbed quantities remain in the frequency domain unless otherwise specified.

\section{B. OWNS equations}

The OWNS parabolization, derived in detail in [27], is now applied to Eq.12] We provide three variations of the parabolization that can be applied under different scenarios as outlined below.

\section{Variation 1: outflow approach}

The outflow approach is appropriate when there is no external forcing $(h=0)$. In addition, streamwise viscous terms must be neglected $(g=0)$ in this version as the parabolization is applied to the inviscid portion of the operator, following the original approach in [27]. This is consistent with the traditional thin-layer approximation invoked in PSE. In the projection approach, discussed in the next section, the need for this approximation is eliminated though we point out that it is highly accurate for the cases considered in this paper. Setting $f=g=0$, we have

$$
\frac{\mathrm{d} \phi}{\mathrm{d} \xi}=M \phi
$$

We can rewrite Eq. 14 by partitioning $M$ as follows

$$
\frac{\mathrm{d}}{\mathrm{d} \xi}\left[\begin{array}{l}
\phi_{+} \\
\phi_{-}
\end{array}\right]=\left[\begin{array}{ll}
M_{++} & M_{+-} \\
M_{-+} & M_{--}
\end{array}\right]\left[\begin{array}{c}
\phi_{+} \\
\phi_{-}
\end{array}\right],
$$

where the,,++--+- , and -+ partitioned blocks are associated with the negative and positive characteristic variables. We now perform an eigenvalue decomposition of $M=V D U$ such that

$$
\begin{gathered}
\phi(\xi, \omega)=V \psi \quad \leftrightarrow \quad \psi=U \phi, \\
\frac{\mathrm{d} \psi}{\mathrm{d} \xi}=D \psi,
\end{gathered}
$$

where the diagonal matrix $D$ contains the eigenvalues, columns of $V$ are the right eigenvectors and rows of $U=V^{-1}$ are the left eigenvectors, all ordered in the same way as the expansion coefficients $\psi$. We can further partition the matrices as follows

$$
\begin{aligned}
\frac{\mathrm{d}}{\mathrm{d} \xi}\left[\begin{array}{l}
\psi_{+} \\
\psi_{-}
\end{array}\right] & =\left[\begin{array}{cc}
D_{++} & 0 \\
0 & D_{--}
\end{array}\right]\left[\begin{array}{l}
\psi_{+} \\
\psi_{-}
\end{array}\right], \\
{\left[\begin{array}{l}
\phi_{+} \\
\phi_{-}
\end{array}\right] } & =\left[\begin{array}{ll}
V_{++} & V_{+-} \\
V_{-+} & V_{--}
\end{array}\right]\left[\begin{array}{l}
\psi_{+} \\
\psi_{-}
\end{array}\right], \\
{\left[\begin{array}{l}
\psi_{+} \\
\psi_{-}
\end{array}\right] } & =\left[\begin{array}{ll}
U_{++} & U_{+-} \\
U_{-+} & U_{--}
\end{array}\right]\left[\begin{array}{l}
\phi_{+} \\
\phi_{-}
\end{array}\right],
\end{aligned}
$$


where $\psi_{+}$and $\psi_{-}$are the downstream and upstream expansion coefficients, respectively. The exact parabolization is

$$
\begin{aligned}
\frac{\mathrm{d} \psi_{+}}{\mathrm{d} \xi} & =D_{++} \psi_{+}, \\
\psi_{-} & =0,
\end{aligned}
$$

or in terms of characteristics with differential-algebraic equation (DAE) system of index 1

$$
\begin{gathered}
\frac{\mathrm{d} \phi_{+}}{\mathrm{d} \xi}=M_{++} \phi_{+}+M_{+-} \phi_{-}, \\
U_{-+} \phi_{+}+U_{--} \phi_{-}=0 .
\end{gathered}
$$

The parabolization is at this point exact, but requires an eigenvalue decomposition of $M$ at each step in order to determine $U_{-+}$and $U_{--}$, which is computationally expensive. However, an excellent approximate parabolization is obtained by designing a high-order recursive filter [27, 31, 32] for these matrices, resulting in the OWNS DAE system

$$
\begin{aligned}
& \frac{\mathrm{d} \phi_{+}}{\mathrm{d} \xi}=M_{++} \phi_{+}+M_{+-} \phi_{-}, \\
& \left(M-i b_{+}^{j} I\right) \phi^{j}=\left(M-i b_{-}^{j} I\right) \phi^{j+1} \quad j=0, \ldots, N_{b}-1, \\
& \phi_{-}^{N_{b}}=0,
\end{aligned}
$$

where $\left\{\phi^{j}: j=0, \ldots, N_{b}\right\}$ and $\left\{b_{+}^{j}, b_{-}^{j}: j=0, \ldots, N_{b}-1\right\}$ are the auxiliary variables and complex-valued recursion parameters, respectively. Note that $N_{b}$ is the order of the approximate one-way system such that as $N_{b} \rightarrow \infty$, we recover the exact parabolization in Eq. 19 [32]. The physical variable $\phi$ is the zero-indexed quantity $\phi^{0}$ and the remaining auxiliary variables are defined via the recursions. The recursion parameters are determined based on the original algorithm described in [27] which derive the spatial eigenvalues of the Euler equations linearized about a uniform flow. These eigenvalues provide a basis which can be used to predict the eigenvalues of the semi-discrete operator $M$ for complicated, non-uniform flows. The DAE system can then be solved using an implicit integration method of choice such as backward differentiation formula (BDF) or implicit Runge-Kutta (RK).

\section{Variation 2: projection approach}

The projection approach alleviates the need to neglect the streamwise viscous terms and the inhomogeneous term. Using the same eigenvalue decomposition as the outflow approach $M=V D U$, we define a projection operator that splits the solution $\phi$ into the right-going and left-going components

$$
P=\left[\begin{array}{ll}
V_{+} & V_{-}
\end{array}\right]\left[\begin{array}{ll}
I & 0 \\
0 & 0
\end{array}\right]\left[\begin{array}{l}
U_{+} \\
U_{-}
\end{array}\right],
$$

such that

$$
\phi_{r}=P \phi, \quad \phi_{l}=(I-P) \phi .
$$

Applying the projection operator to Eq. 12 yields

$$
P \frac{\mathrm{d} \phi}{\mathrm{d} \xi}=P(M \phi+h+g)
$$

and the fact that $P$ and $M$ commute we write [32]

$$
\frac{\mathrm{d} \phi_{r}}{\mathrm{~d} \xi}=P\left(M \phi_{r}+h+g\right)
$$


As with the outflow approach, we again use a high-order recursive filter to generate an approximate projection operator $\widetilde{P}[32]$. Specifically, the following recursions are used to determine $\widetilde{P}$ acting on an arbitrary vector $\phi$

$$
\begin{array}{ll}
\phi_{+}^{-N_{b}}=0, & \\
\left(M-i b_{-}^{j} I\right) \phi^{-j}-\left(M-i b_{+}^{j} I\right) \phi^{-(j+1)}=0 & j=1, \ldots, N_{b}-1, \\
\left(M-i b_{-}^{0} I\right) \phi^{0}-\left(M-i b_{+}^{0} I\right) \phi^{-1}=\left(M-i b_{-}^{0} I\right) \phi, & \\
\left(M-i b_{+}^{j} I\right) \phi^{j}-\left(M-i b_{-}^{j} I\right) \phi^{(j+1)}=0 & j=0, \ldots, N_{b}-1, \\
\phi_{-}^{N_{b}}=0 . &
\end{array}
$$

The above equations in conjunction with Eq.25 form a DAE system. However, the integration method has an impact on the streamwise viscous terms, which must be treated explicitly and where the streamwise derivatives are approximated with second-order finite differences. The remaining terms, on the other hand, require an implicit integration and thus the stepper must be of a split implicit-explicit (IMEX) type. We have found that an IMEX-BDF scheme is the most efficient choice meeting these requirements.

Despite the projection approach having the obvious benefit of including an arbitrary forcing function and streamwise viscous terms, this approach involves approximately 3 times more operations than the outflow approach [32].

\section{Variation 3: hybrid approach}

A further computational advantage can be obtained by adopting the implicit-explicit splitting scheme applied to the projection method, but including in the explicit part the inhomogeneous forcing term $(h)$. In this way, the projection operator only need be applied to the explicit right-hand-side terms at each integration step. We illustrate this by discretizing Eq. 12 using an $s^{\text {th }}$ order IMEX-BDF scheme

$$
\phi^{i+1}=\sum_{n=1}^{s} \alpha_{n} \phi^{i+1-n}+d \xi \beta_{1}\left(M^{i+1} \phi^{i+1}+h^{i+1}\right)+d \xi \sum_{n=1}^{s} \gamma_{n} g^{i+1-n},
$$

where $\alpha_{n}, \beta_{1}$, and $\gamma_{n} \in \mathbb{R}$. We can rearrange the equation as follows

$$
\phi^{i+1}=\left(I-d \xi \beta_{1} M^{i+1}\right)^{-1} \sum_{n=1}^{s} \alpha_{n} \phi^{i+1-n}+\left(I-d \xi \beta_{1} M^{i+1}\right)^{-1} d \xi\left(\beta_{1} h^{i+1}+\sum_{n=1}^{s} \gamma_{n} g^{i+1-n}\right) .
$$

Since Eq. 12 is a linear inhomogeneous ODE, we can solve the homogeneous and particular solutions separately as follows

$$
\begin{gathered}
\phi_{h}^{i+1}=\left(I-d \xi \beta_{1} M^{i+1}\right)^{-1} \sum_{n=1}^{s} \alpha_{n} \phi^{i+1-n} \\
\phi_{p}^{i+1}=\left(I-d \xi \beta_{1} M^{i+1}\right)^{-1} d \xi\left(\beta_{1} h^{i+1}+\sum_{n=1}^{s} \gamma_{n} g^{i+1-n}\right)
\end{gathered}
$$

Note that $h^{i+1}$ and $M^{i+1}$ are known quantities. Up until now, we have only discretized the exact characteristic equation - no approximations have been made. However, if we want to only solve for the right-going solution, we can apply the OWNS approximations and solve the homogeneous solution via the outflow approach and apply the approximate projection operator $\widetilde{P}$ to the particular solution yielding

$$
\begin{gathered}
\phi_{h}^{i+1} \approx \phi_{\text {outflow }}^{i+1}, \\
\phi_{p}^{i+1} \approx \widetilde{P} \phi_{p}^{i+1}, \\
\phi^{i+1} \approx \phi_{\text {outflow }}^{i+1}+\widetilde{P} \phi_{p}^{i+1} .
\end{gathered}
$$


Using this approach, the only DAE system solved is that of the outflow method which is computationally cheaper since about twice as many auxiliary variables $\phi^{j}$ are required at a given order $N_{b}$ for the projection method [32].

We also note that including the streamwise viscous terms can occasionally lead to numerical instability. Specifically, we must have a sufficiently large local grid Reynolds number $R e_{g}=\frac{\bar{\rho}^{*} \bar{u}_{\xi}^{*} d \xi^{*}}{\bar{\mu}^{*}}$ or Peclet number $P e_{g}=R e_{g} P r$, which essentially requires the base flow to be advection dominated. Should numerical instability occur, either the streamwise grid size $d \xi$ can be increased or the streamwise viscous terms can be neglected altogether, which is reasonable since high-speed boundary layers are relatively thin $\left(\delta^{*} \sim u_{\infty}^{*}-1 / 2\right.$ for laminar flow).

\section{Equation of state}

The OWNS methodology assumes the fluid to be an ideal gas with $c_{v}=c_{v}(T)$ and $c_{p}=c_{p}(T)$, and fluid properties $k, \mu, \kappa$, and $\gamma$ that depend solely on temperature. We denote any of the aforementioned fluid properties as $\Phi$ and perform a Taylor series expansion about $\bar{T}$

$$
\Phi(T)=\Phi(\bar{T})+\left.\frac{d \Phi}{d T}\right|_{T=\bar{T}} T^{\prime}+\text { higher order terms. }
$$

The linearized fluid property perturbation is thus

$$
\Phi^{\prime}=\left.\frac{d \Phi}{d T}\right|_{T=\bar{T}} T_{l}^{\prime},
$$

where $T_{l}^{\prime}$ is the temperature perturbation from the linearized equation of state

$$
T_{l}^{\prime}=\frac{\bar{p} v^{\prime}+\bar{v} p^{\prime}}{R}
$$

For the validation cases presented in the next section, we are comparing to other numerical solutions where a calorically perfect gas (constant specific heats) has been assumed. Thus, unless otherwise stated, we likewise assume the fluid as calorically perfect air with $\gamma=1.4$ and $P r=0.72$ and, moreover, viscosity and thermal conductivity are calculated using Sutherland's law

$$
\mu^{*}=\mu_{\infty}^{*}\left(\frac{T^{*}}{T_{\infty}^{*}}\right)^{3 / 2} \frac{T_{\infty}^{*}+S^{*}}{T^{*}+S^{*}}
$$

where $S^{*}=110.4 \mathrm{~K}$.

\section{Computational setup}

To properly resolve the instabilities near the wall and critical layer, we employ a grid stretching technique [33] for all validation cases

$$
\begin{aligned}
\eta & =\frac{a \lambda}{b-\lambda}, \\
b & =1+a / \eta_{\max }, \\
a & =\eta_{\max } \eta_{i} /\left(\eta_{\max }-2 \eta_{i}\right),
\end{aligned}
$$

where $0 \leq \lambda \leq 1$ (equidistant grid points). Using the above scheme, half the points in the $\eta$ direction are placed between $\eta=0$ and $\eta=\eta_{i}$.

Details of each validation case are outlined below. Noting that all validation cases have no external forcing $(h=0)$ and since they are all studied at hypersonic speeds with high $R e_{\infty}^{*}$, the thin-shear-layer approximation is made $(g \approx 0)$. Thus, the outflow approach is used for all OWNS computations with second-order BDF (BDF2) spatial integration. Additionally, this paper will focus strictly on two-dimensional instabilities, i.e. $\beta=0$.

\section{Adiabatic flat plate}

We first validate OWNS by comparing to DNS of a two-dimensional Mach 4.5 adiabatic-wall, flat-plate boundary layer [25]. The parameters are given in Table 1. The base flow is computed using the Howarth-Dorodnitsyn transformation of the compressible Blasius equations under the assumption $d \bar{p}=0$. Note that $\gamma$ was not explicitly 
provided in [25] so a value of 1.4 was assumed. The computational domain is summarized in Table 2 where $\xi$ and $\eta$ coincide with the global $x$ and $y$ axes with its origin placed at the plate leading edge.

Table 1 Adiabatic flat-plate flow parameters from [25].

\begin{tabular}{ccccc}
\hline$M_{\infty}$ & $T_{\infty}^{*}[\mathrm{~K}]$ & $T_{w}^{*}[\mathrm{~K}]$ & $p_{\infty}^{*}[\mathrm{~Pa}]$ & $R e_{\infty}^{*}[1 / \mathrm{m}]$ \\
\hline 4.5 & 65.15 & $T_{a d}^{*}$ & 728.438 & $7.2 \times 10^{6}$ \\
\hline
\end{tabular}

Table 2 Computational domain used for adiabatic flat-plate stability analyses.

\begin{tabular}{cccccc}
\hline$\xi_{\min }^{*}[\mathrm{~m}]$ & $\xi_{\max }^{*}[\mathrm{~m}]$ & $\eta_{\min }^{*}[\mathrm{~m}]$ & $\eta_{\max }^{*}[\mathrm{~m}]$ & $N_{\xi}$ & $N_{\eta}$ \\
\hline 0.025 & 0.63 & 0.0 & 0.0118 & 8000 & 800 \\
\hline
\end{tabular}

The self-similar base flow solutions for $\frac{\bar{u}_{\xi}^{*}}{u_{\infty}^{*}}$ and $\frac{\bar{T}^{*}}{T_{\infty}^{*}}$ are presented in Figure 1 . The similarity variable $\chi$ is defined as

$$
\chi=\sqrt{\frac{u_{\infty}^{*} \rho_{\infty}^{*}}{2 \mu_{\infty}^{*} \xi^{*}}} \int_{0}^{\eta^{*}} \frac{\rho^{*}}{\rho_{\infty}^{*}} d \eta^{*} .
$$

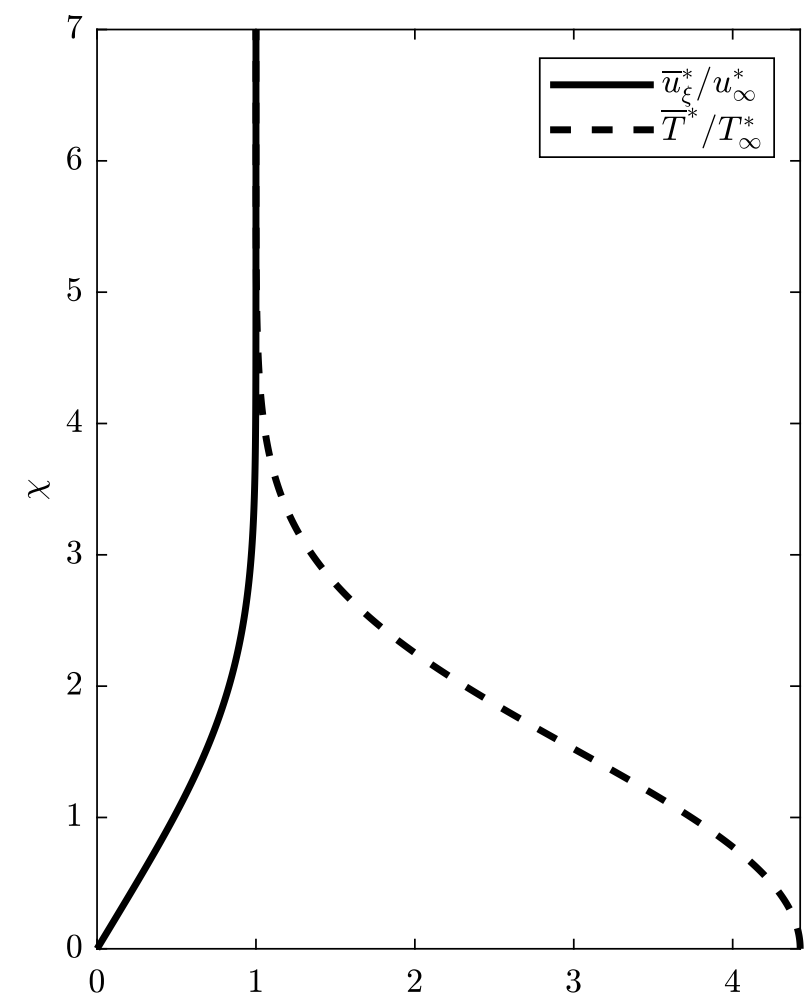

Fig. 1 Self-similar base flow solutions $\frac{\bar{u}_{\xi}^{*}}{u_{\infty}^{*}}$ and $\frac{\bar{T}^{*}}{T_{\infty}^{*}}$ for Case 1.

\section{Highly-cooled (isothermal) flat plate}

The second validation case is also a flat-plate configuration but with a cold wall [24]. Parameters are given in Table 3 . Similar to the adiabatic case, the base flow is computed using the Howarth-Dorodnitsyn transformation of the 
compressible Blasius equations under the assumption $d \bar{p}=0$. The computational domain is summarized in Table 4 where $\xi$ and $\eta$ coincide with the global $x$ and $y$ axes with its origin placed at the plate leading edge.

Table 3 Highly-cooled flat-plate flow parameters from $[24]$. Note that $L^{*}=0.0382 \mathrm{~m}$.

\begin{tabular}{ccccc}
\hline$M_{\infty}$ & $T_{\infty}^{*}[\mathrm{~K}]$ & $T_{w}^{*}[\mathrm{~K}]$ & $p_{\infty}^{*}[\mathrm{kPa}]$ & $R e=\frac{\rho_{\infty}^{*} u_{\infty}^{*} L^{*}}{\mu_{\infty}^{*}}$ \\
\hline 6.0 & 300 & 150 & 20 & $10^{6}$ \\
\hline
\end{tabular}

Table 4 Computational domain used for highly-cooled flat-plate stability analyses.

\begin{tabular}{ccccccc}
\hline$F$ & $\xi_{\min }^{*}[\mathrm{~m}]$ & $\xi_{\max }^{*}[\mathrm{~m}]$ & $\eta_{\min }^{*}[\mathrm{~m}]$ & $\eta_{\max }^{*}[\mathrm{~m}]$ & $N_{\xi}$ & $N_{\eta}$ \\
\hline $1.3124 \cdot 10^{-4}$ & 0.0956 & 0.2677 & 0.0 & 0.0574 & 8000 & 1000 \\
$2.0590 \cdot 10^{-4}$ & 0.0388 & 0.1721 & 0.0 & 0.0367 & 8000 & 1000 \\
\hline
\end{tabular}

The self-similar base flow solutions for $\frac{\bar{u}_{\xi}^{*}}{u_{\infty}^{*}}$ and $\frac{\bar{T}^{*}}{T_{\infty}^{*}}$ are presented in Figure 2 . The similarity variable $\chi$ is the same as the adiabatic flat-plate case.

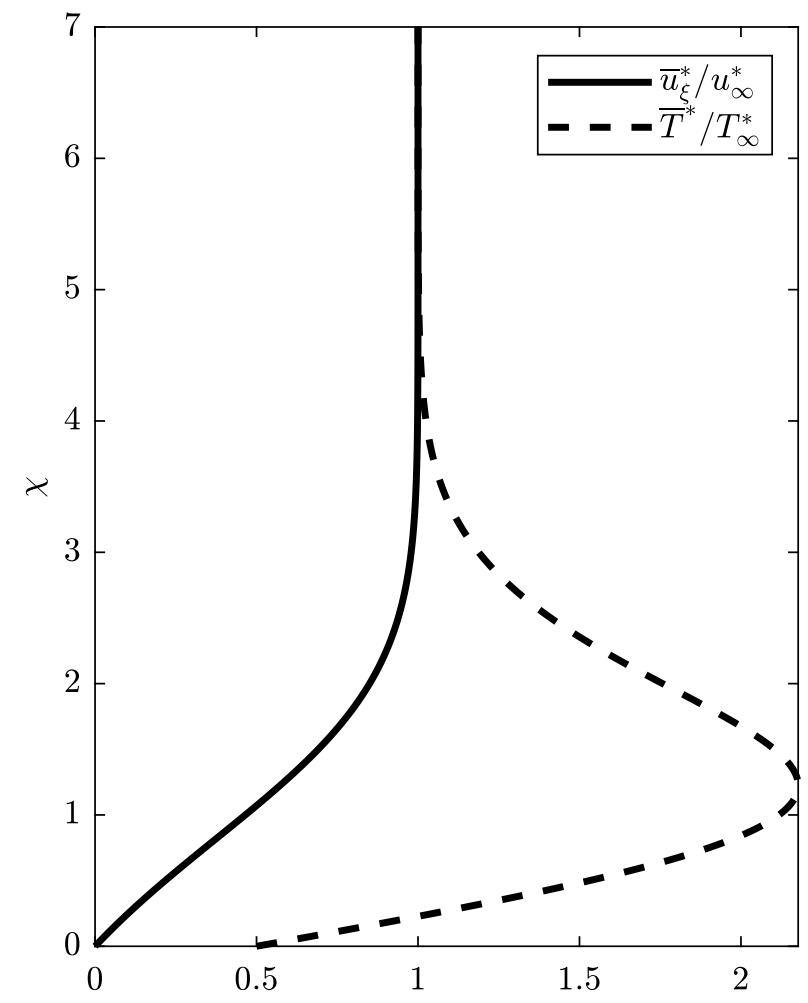

Fig. 2 Self-similar base flow solutions $\frac{\bar{u}_{\xi}^{*}}{u_{\infty}^{*}}$ and $\frac{\bar{T}^{*}}{T_{\infty}^{*}}$ for Case 2.

\section{Isothermal sharp cone}

The third case we consider is an axisymmetric 7-deg half-angle sharp cone [34] with the flow parameters based on tests performed by Wagner [35] in the DLR High Enthalpy Shock Tunnel Göttingen (HEG) and given in Table 5. An inviscid solution of the base flow was first determined by solving the Taylor-Maccoll ODE [36] using a modified MATLAB code from [37]. Thereafter, the compressible Blasius boundary layer was computed by applying the Mangler 
transformation [38] for axisymmetric flows [39]. Note that $P r=0.704$ for this case and although not explicitly mentioned in [34], $\gamma$ was assumed to be 1.4 and the viscosity is chosen by satisfying Sutherland's law (Eq. 37) with $R e_{e}^{*}=5.69 \times 10^{6} \mathrm{~m}^{-1}$. The computational domain is summarized in Table 6 in which the $\xi$ and $\eta$ axes originate from the cone nose.

Table 5 Sharp-cone flow parameters from [34].

\begin{tabular}{cccccc}
\hline$M_{\infty}$ & $T_{\infty}^{*}[\mathrm{~K}]$ & $T_{w}^{*}[\mathrm{~K}]$ & $p_{\infty}^{*}[\mathrm{~Pa}]$ & $R e_{\infty}^{*}[1 / \mathrm{m}]$ & $f^{*}[\mathrm{kHz}]$ \\
\hline 7.4 & 268 & 300 & 2129 & $4.06 \times 10^{6}$ & 550 \\
\hline
\end{tabular}

Table 6 Computational domain used for sharp-cone stability analyses.

\begin{tabular}{cccccc}
\hline$\xi_{\min }^{*}[\mathrm{~m}]$ & $\xi_{\max }^{*}[\mathrm{~m}]$ & $\eta_{\min }^{*}[\mathrm{~m}]$ & $\eta_{\max }^{*}[\mathrm{~m}]$ & $N_{\xi}$ & $N_{\eta}$ \\
\hline 0.14 & 0.95 & 0.0 & 0.0186 & 8294 & 400 \\
\hline
\end{tabular}

The self-similar base flow solutions for $\frac{\bar{u}_{\xi}^{*}}{u_{e}^{*}}$ and $\frac{\bar{T}^{*}}{T_{e}^{*}}$ are presented in Figure 3 The similarity variable $\chi$ is defined as

$$
\chi=\sqrt{\frac{3 u_{e}^{*} \rho_{e}^{*}}{2 \mu_{e}^{*} \xi^{*}}} \int_{0}^{\eta^{*}} \frac{\rho^{*}}{\rho_{e}^{*}} d \eta^{*} .
$$

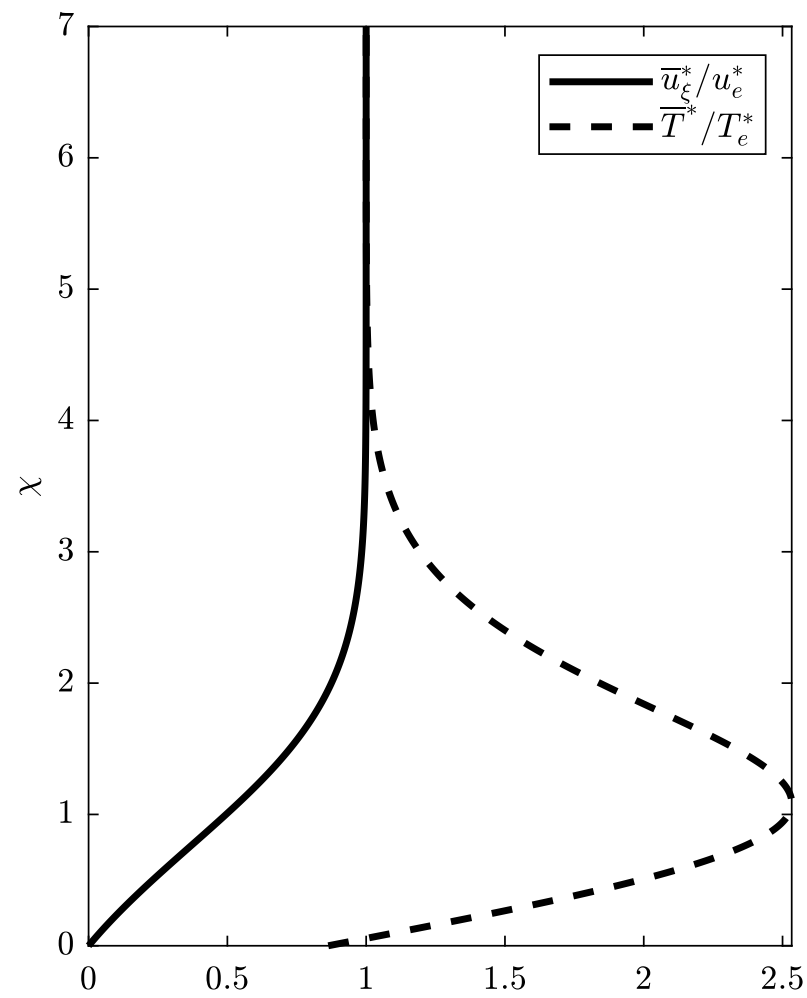

Fig. 3 Self-similar base flow solutions $\frac{\bar{u}_{\xi}^{*}}{u_{e}^{*}}$ and $\frac{\bar{T}^{*}}{T_{e}^{*}}$ for Case 3. 


\section{HIFiRE-5 elliptic cone}

The final validation case is a $38.1 \%$ scale model of the HIFiRE-5 elliptic cone with an axial length of $38.1 \mathrm{~mm}$ and an aspect ratio of 2:1 at the tip. The cone half-angles are 7.00 and 13.797 degrees along the minor (centerline) and major (attachment line) axes, respectively. The base flow is provided by The Boeing Company with the freestream parameters summarized in Table 7. We focus our study on the centerline, and for validation purposes treat the flow and disturbances there as locally two-dimensional and compare with solutions found using LASTRAC [40]. The streamwise computational domain varies slightly depending on the frequency analyzed and is summarized in Table 8 . The wall-normal domain extends from the cone surface to the bow shock. For all LST computations, we have $N_{\xi} \times N_{\eta}=100 \times 600$ and for the OWNS computations we have $N_{\xi} \times N_{\eta}=5000 \times 600$. The origin of the curvilinear coordinates correspond to $x^{*}=8.364 \cdot 10^{-4} \mathrm{~m}$ and $y^{*}=9.454 \cdot 10^{-4} \mathrm{~m}$ (global $x$ and $y$ axes originate from the cone nose).

Table 7 HIFiRE-5 flow parameters.

\begin{tabular}{ccccc}
\hline$M_{\infty}$ & $T_{\infty}^{*}[\mathrm{~K}]$ & $T_{w}^{*}[\mathrm{~K}]$ & $p_{\infty}^{*}[\mathrm{~Pa}]$ & $R e_{\infty}^{*}[1 / \mathrm{m}]$ \\
\hline 6.0 & 49.45 & 315 & 417.315 & $8.1 \times 10^{6}$ \\
\hline
\end{tabular}

Table 8 Shock-fitted computational domain used for HIFiRE-5 stability analyses.

\begin{tabular}{ccc}
\hline$f^{*}[\mathrm{kHz}]$ & $\xi_{\text {min }}^{*}[\mathrm{~m}]$ & $\xi_{\text {max }}^{*}[\mathrm{~m}]$ \\
\hline 60 & 0.101 & 0.305 \\
70 & 0.0807 & 0.305 \\
80 & 0.0652 & 0.305 \\
\hline
\end{tabular}

Contour plots of $\bar{u}_{x}^{*}$ and $\bar{T}^{*}$ acquired from The Boeing Company along the centerline of the HIFiRE-5 elliptic cone is shown in Figure 4. 

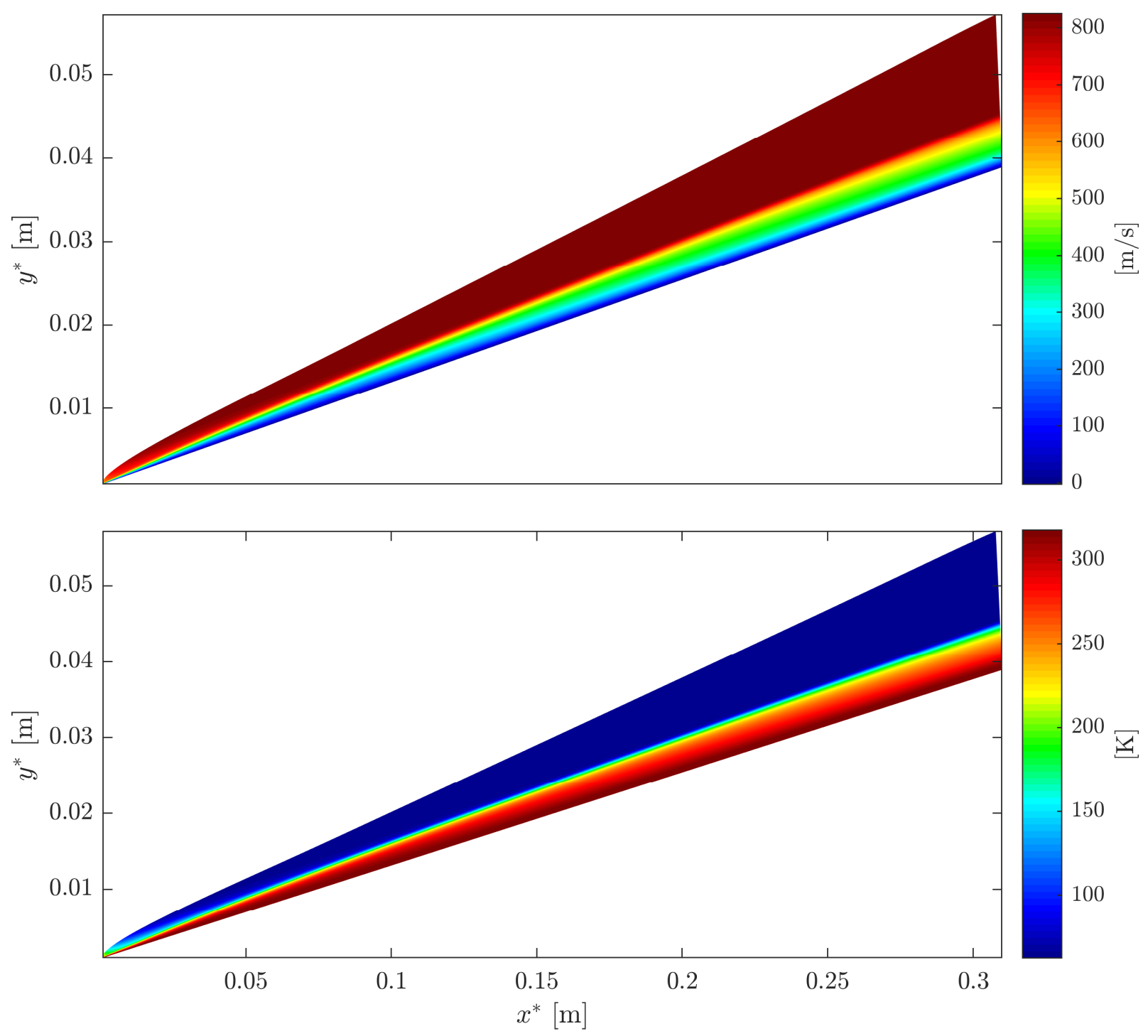

Fig. 4 DNS-computed centerline base flow solutions $\bar{u}_{x}^{*}$ (top) and $\bar{T}^{*}$ (bottom) from The Boeing Company for Case 4.

\section{E. Boundary conditions}

No-slip boundary conditions $\left(u^{\prime}=v^{\prime}=0\right)$ are imposed at the wall along with $T^{\prime}=0$. The latter condition is enforced because Mack [4] and Malik [33] argued that for high-frequency disturbances, the thermal inertia of the body will preclude temperature perturbations from penetrating deep into the solid boundary. Thus, at the wall, we solve the continuity and linearized equation of state for $p^{\prime}$ and $v^{\prime}$. At the upper boundary, we impose 1D inviscid Thompson characteristic boundary conditions [41] to prevent spurious numerical reflections. The inviscid assumption is valid at the upper boundary since viscous effects from the boundary layer are negligible in this region. The OWNS marches are initialized with the right-going component of eigenfunctions from quasi-parallel LST corresponding to a specific mode at the inlet. 


\section{Results}

\section{A. Adiabatic flat plate}

We initialize the OWNS march at the inlet with the first mode, i.e. Mode $\mathrm{S}$, with $N_{b}=20$. Refer to [19] for terminology regarding the slow (Mode S) and fast (Mode F1, Mode F2, etc.) acoustic modes. Figures 5 and 6 compare the wavenumber $\alpha_{r}$, the disturbance growth rate $\alpha_{i}$, and the wall-pressure perturbations $\left|p_{w}^{\prime}\right|$ from OWNS and DNS at $F=2.2 \cdot 10^{-4}$. The wavenumber and growth rate are defined as [25]

$$
\begin{gathered}
\alpha_{r}=\frac{d\left|p_{w_{\theta}}^{\prime}\right|}{d \xi}, \\
\alpha_{i}=-\frac{1}{\left|p_{w}^{\prime}\right|} \frac{d\left|p_{w}^{\prime}\right|}{d \xi},
\end{gathered}
$$

where $p_{w_{\theta}}^{\prime}$ is the phase angle of the wall-pressure perturbation. Note that if multiple modes are present, we will observe the modulated effect of these waves in $\alpha_{r}$ and $\alpha_{i}$. 


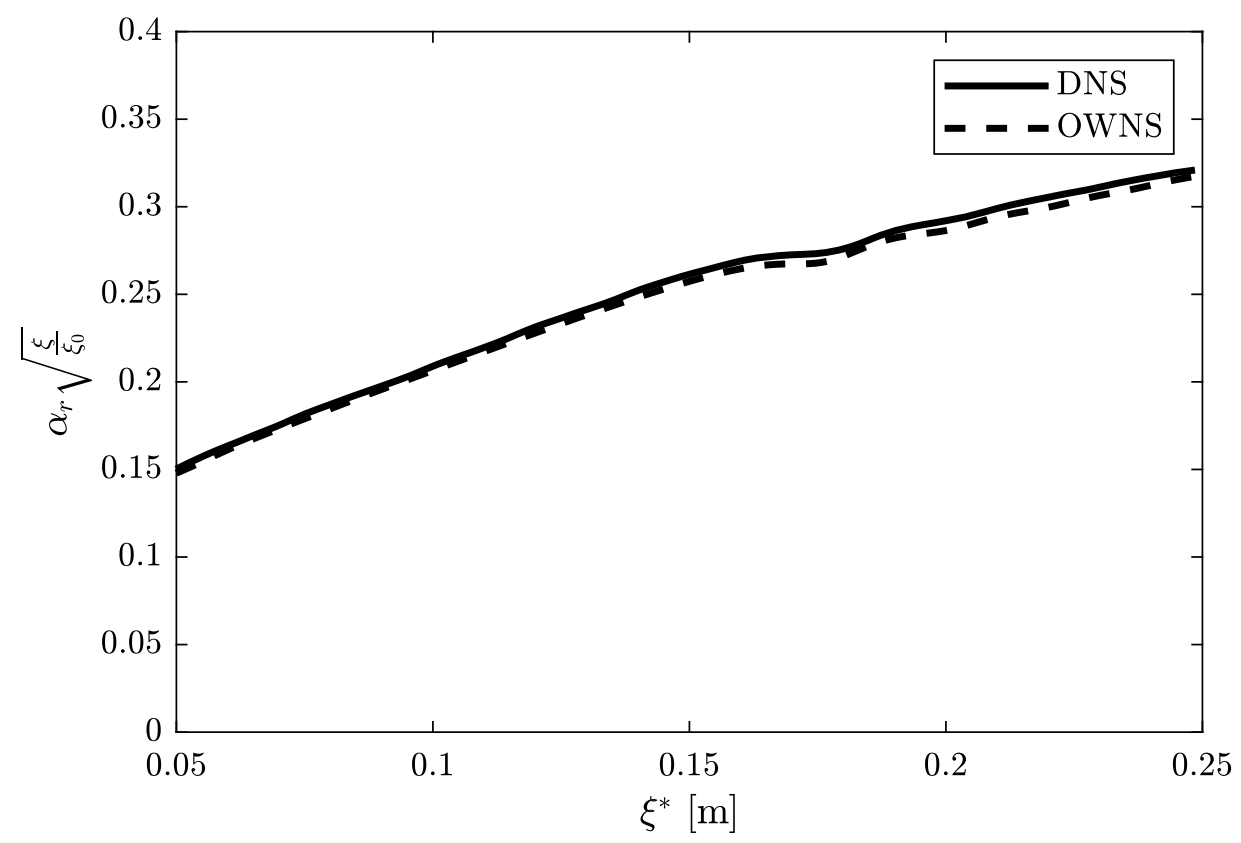

(a) Scaled streamwise wavenumber $\alpha_{r} \sqrt{\frac{\xi}{\xi_{0}}}$.

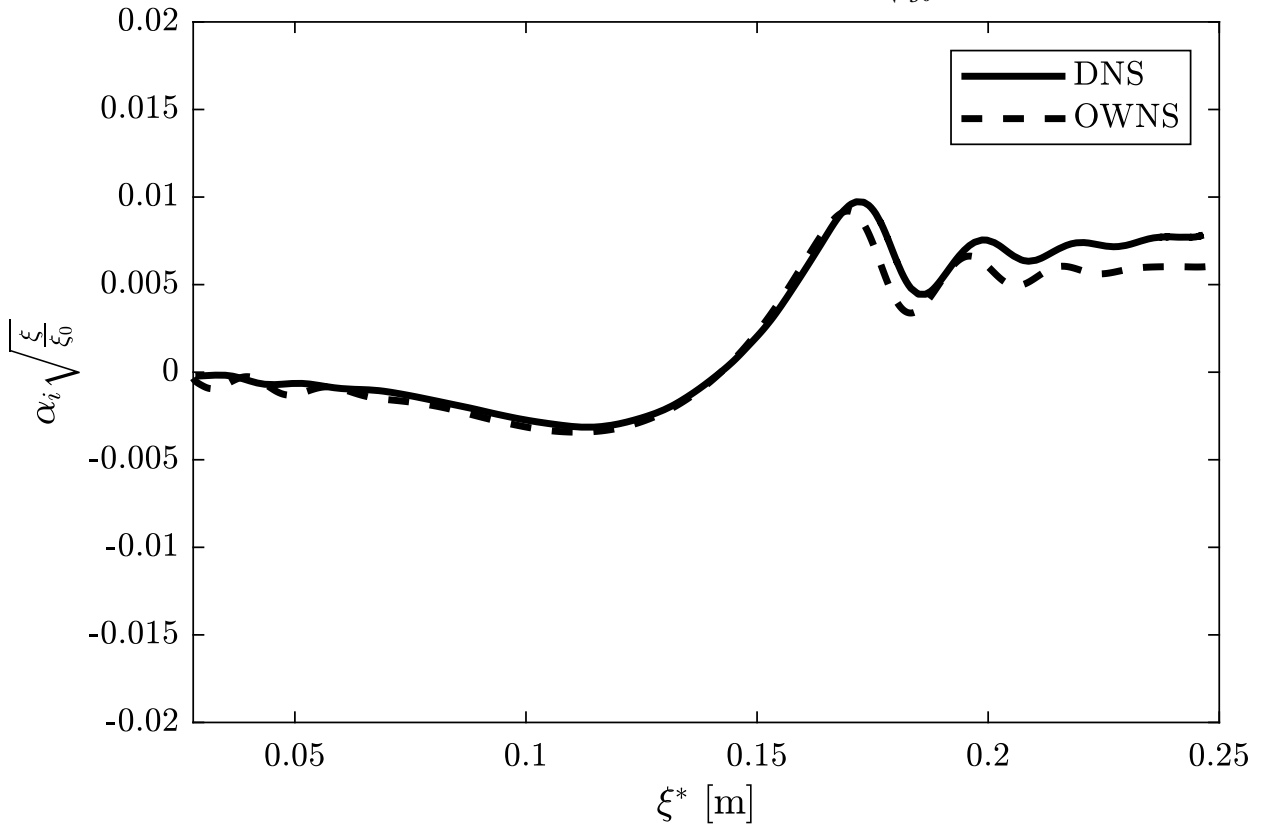

(b) Scaled growth rate $\alpha_{i} \sqrt{\frac{\xi}{\xi_{0}}}$.

Fig. 5 Streamwise wavenumber (a) and growth rate (b) between OWNS and DNS [25] when Mode S is initialized at the inlet. 


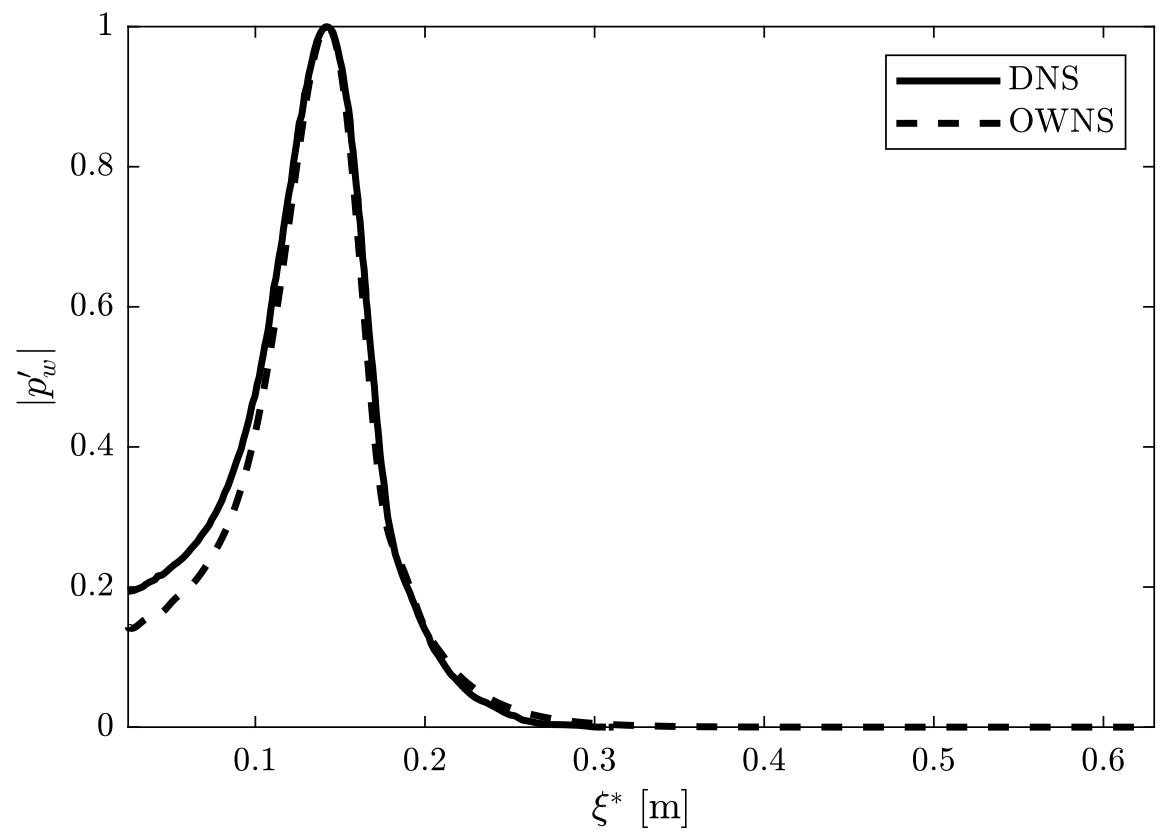

Fig. 6 Wall-pressure perturbations from DNS [25] and OWNS with arbitrary linear scale applied.

When the unstable Mode $\mathrm{S}$ is initialized at $\xi^{*}=0.025 \mathrm{~m}$, there is also the presence of the stable Mode F1 causing the small oscillation in growth rate. Although it cannot be observed in Figures 5 or 6, LST results from [25] indicate synchronization of these two modes at $\xi^{*} \approx 0.098 \mathrm{~m}$, in which then Mode $\mathrm{S}$ becomes the canonical second Mack mode due to resonant interactions with the stable Mode F1. This resonance continues until $\xi^{*} \approx 0.142 \mathrm{~m}$ which is the neutral stability point of the second mode after which the wall-pressure perturbations decay rapidly to zero. The oscillation in growth rate after the neutral stability point is due to the presence of the now stable second mode, Mode F1, and other higher-order fast acoustic modes. Overall, we observe excellent agreement between OWNS and DNS in the wall-pressure distribution and streamwise wavenumber. Although there are small discrepancies in the growth rates, both methods yielded a second-mode neutral stability point of $\xi^{*} \approx 0.142 \mathrm{~m}$.

\section{B. Highly-cooled (isothermal) flat plate}

To compare results with [24], we non-dimensionalize $\xi^{*}$ and $\eta^{*}$ with $L^{*}=0.0382 \mathrm{~m}$, i.e. the distance from the plate leading edge. We will first study the low-frequency (LF) wave train at $F=1.3124 \cdot 10^{-4}$ which corresponds to Mode F1 originating from the branch point $c_{F}=M_{\infty}+1$ of the fast acoustic waves. We thus initialize the OWNS march with this mode at the inlet with $N_{b}=20$. Figure 7 shows the wall-pressure distribution and the pressure disturbance field computed with OWNS. In accordance to LST from [24], the inlet disturbances grow exponentially in the boundary layer until $\xi_{k} \approx 4.5$ where the second mode begins to synchronize with the slow acoustic branch. At this point, a new discrete stable mode is also formed. From $4.5<\xi<5.6$, the second mode radiates slow acoustic waves and generates the outer acoustic field seen in Figure 7 . At $\xi_{s} \approx 5.6$, the second mode coalesces with the slow acoustic branch. We note that the spontaneous radiation of sound is localized to this region [24]. The complex pattern observed below the main beam is attributed to interactions between monochromatic waves having different amplitudes and front angles [24]. Referring to Figure 8 from [24], there is excellent agreement between DNS and OWNS which demonstrates OWNS' capability of accurately capturing the complete disturbance evolution at a fraction of the cost of DNS. 

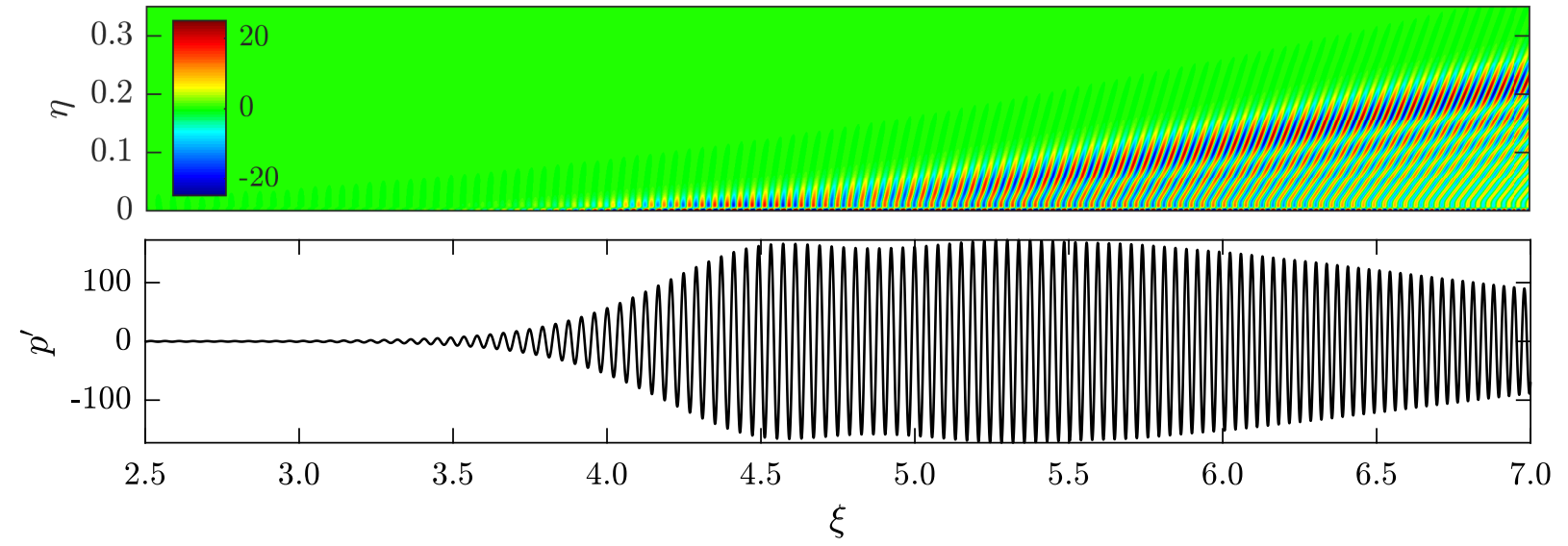

(a) Pressure disturbance field (top) and wall-pressure distribution (bottom).

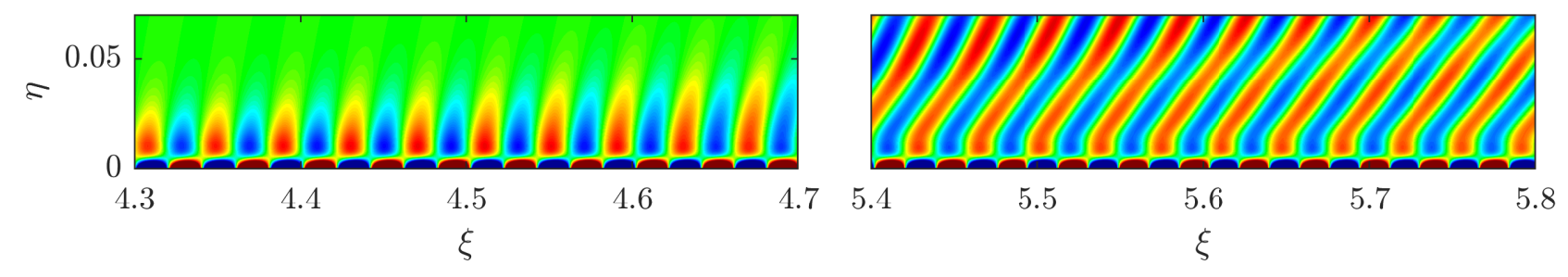

(b) Start of synchronization region at $\xi_{k} \approx 4.5$ (left) and point of coalescence at $\xi_{s} \approx 5.6$ (right).

Fig. 7 OWNS results for $\mathrm{LF}$ wave train at $F=1.3124 \cdot 10^{-4}$ for highly-cooled flat plate. Pressure disturbance field and wall-pressure distribution (a) and close-up of start of synchronization region and point of coalescence (b).

We now study the high-frequency (HF) wave train at $F=2.0590 \cdot 10^{-4}$ which corresponds to the mode originating near the branch point $c_{S}=M_{\infty}-1$ of the slow acoustic waves. The OWNS march is therefore initialized with this mode at the inlet with $N_{b}=20$. Figure 8 shows the wall-pressure distribution and the pressure disturbance field calculated using OWNS. LST results from [24] indicate that this mode becomes unstable as it propagates downstream before becoming stable and having its phase speed drop below $c_{S}$. In contrast to the LF case, the second mode does not coalesce with the slow acoustic branch but is in close proximity before becoming stable. In the vicinity of $c_{S}$, the second mode radiates slow acoustic waves at $\xi_{s} \approx 1.9$ as observed in Figure 8 However, the outer acoustic field is much narrower and weaker than the LF case which is corroborated with LST and DNS findings of [24]. Below the main beam, the acoustic pattern is emblematic of slow acoustic waves being radiated by a stationary local harmonic source which is hypothesized to occur due to the spatial inhomogeneity of the region near $\xi_{s} \approx 1.9$ [24]. Referring to Figure 11 from [24], we again observe excellent agreement between DNS and OWNS. 

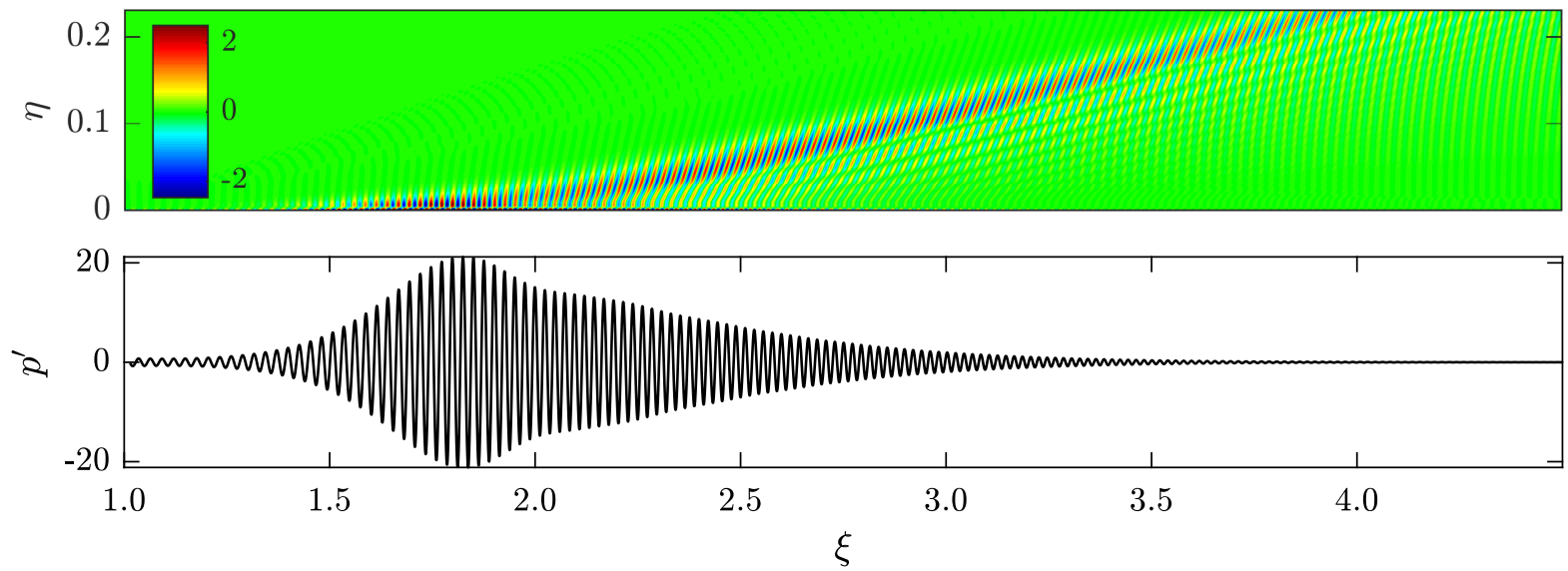

(a) Pressure disturbance field (top) and wall-pressure distribution (bottom).
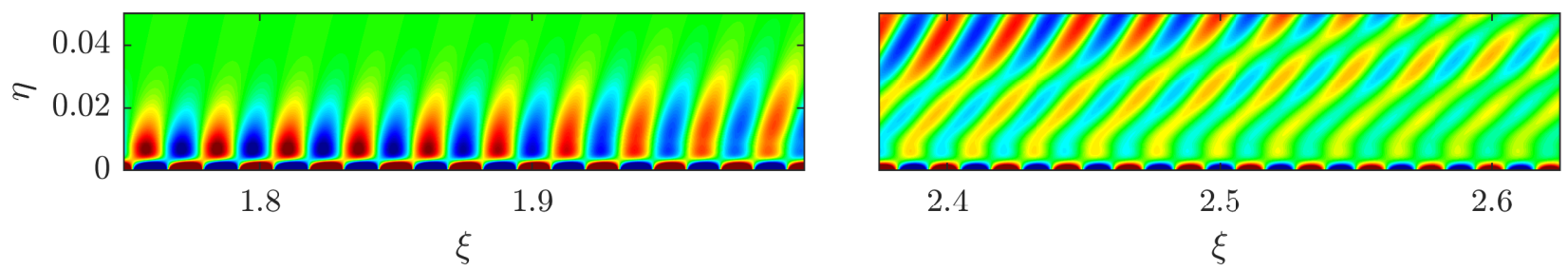

(b) Start of spontaneous acoustic radiation at $\xi_{s} \approx 1.9$ (left) and downstream region (right).

Fig. 8 OWNS results for HF wave train at $F=2.0590 \cdot 10^{-4}$ for highly-cooled flat plate. Pressure disturbance field and wall-pressure distribution (a) and close-up of when the second mode begins to radiate slow acoustic waves at $\xi_{s} \approx 1.9$ and downstream region (b).

\section{Isothermal sharp cone}

We first initialize the OWNS march with Mode $\mathrm{S}$ at the inlet with $N_{b}=12$. Wall-pressure perturbations from OWNS and DNS [34] at the conditions prescribed in Table 5 are compared in Figure 9

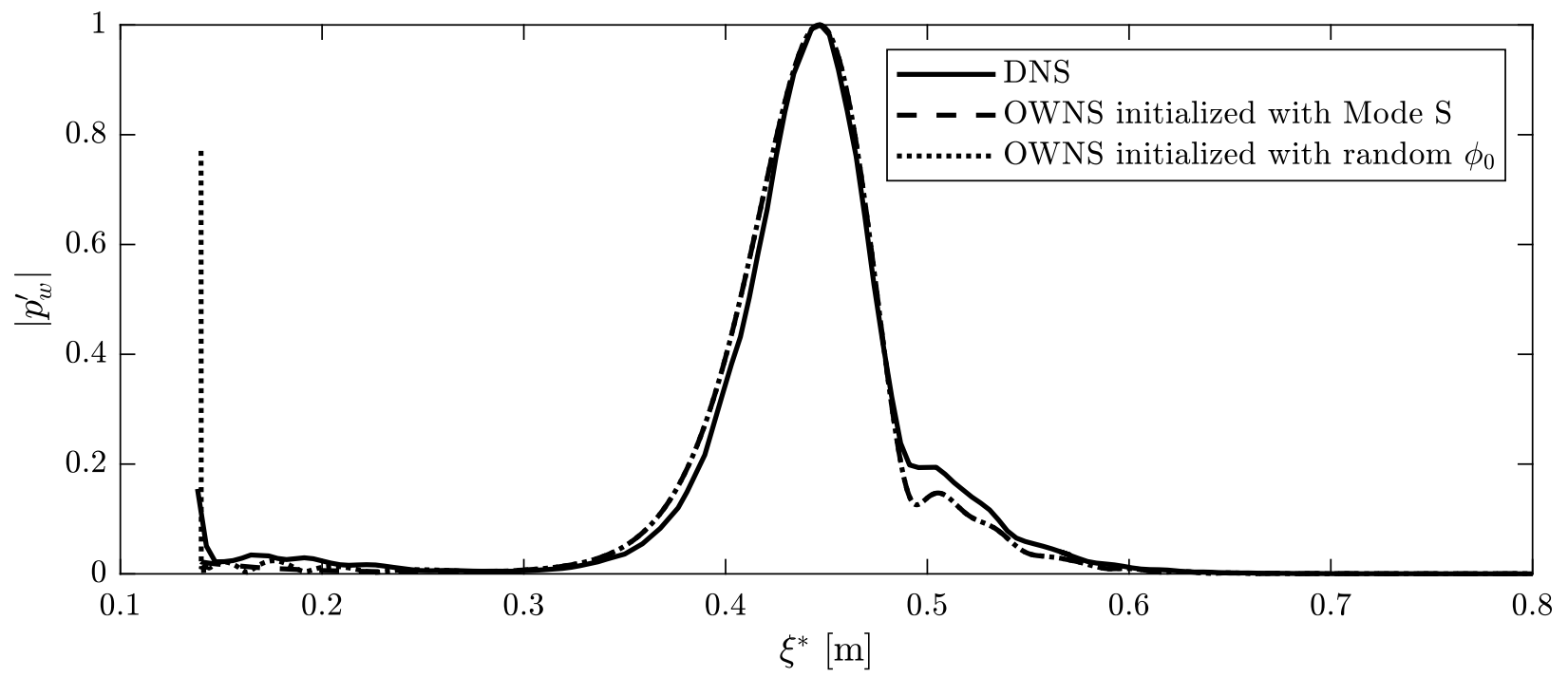

Fig. 9 Wall-pressure perturbations between OWNS and DNS [34] with different initial conditions at $R e_{\infty}=$ $4.06 \times 10^{6} \mathrm{~m}^{-1}$ and $f=550 \mathrm{kHz}$. Arbitrary linear scale applied. 
Overall, we see excellent agreement between the OWNS and DNS wall-pressure signal. Although not apparent from Figure 9 . LST results indicate that the unstable Mode S (first mode) synchronizes with the stable Mode F1 at $\xi^{*} \approx 0.323$ $\mathrm{m}$ and the former becomes the second Mack mode. At $\xi^{*} \approx 0.447 \mathrm{~m}$, the second mode reaches its neutral stability point and the resonant interactions with Mode F1 cease and the wall-pressure disturbances decay rapidly. However, we observe a kink in the wall-pressure profile during the decay. To understand why this occurs, we must gain a better insight into how the modes interact spatially, and thus we plot the specific volume disturbance field in Figure 10.

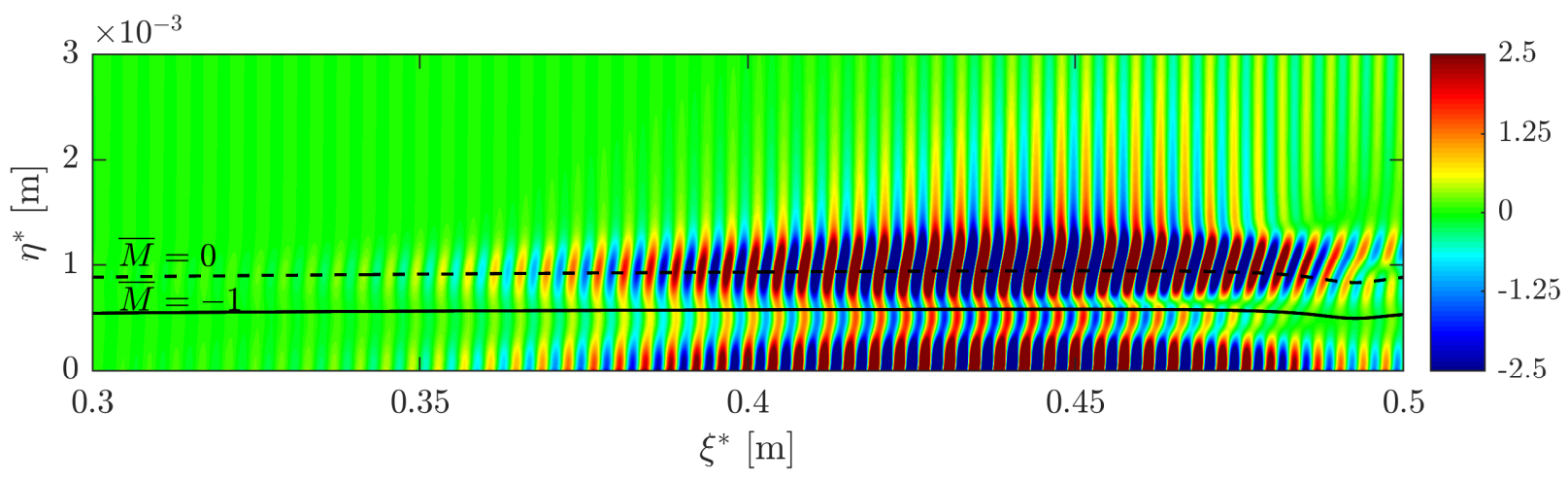

(a) Second mode

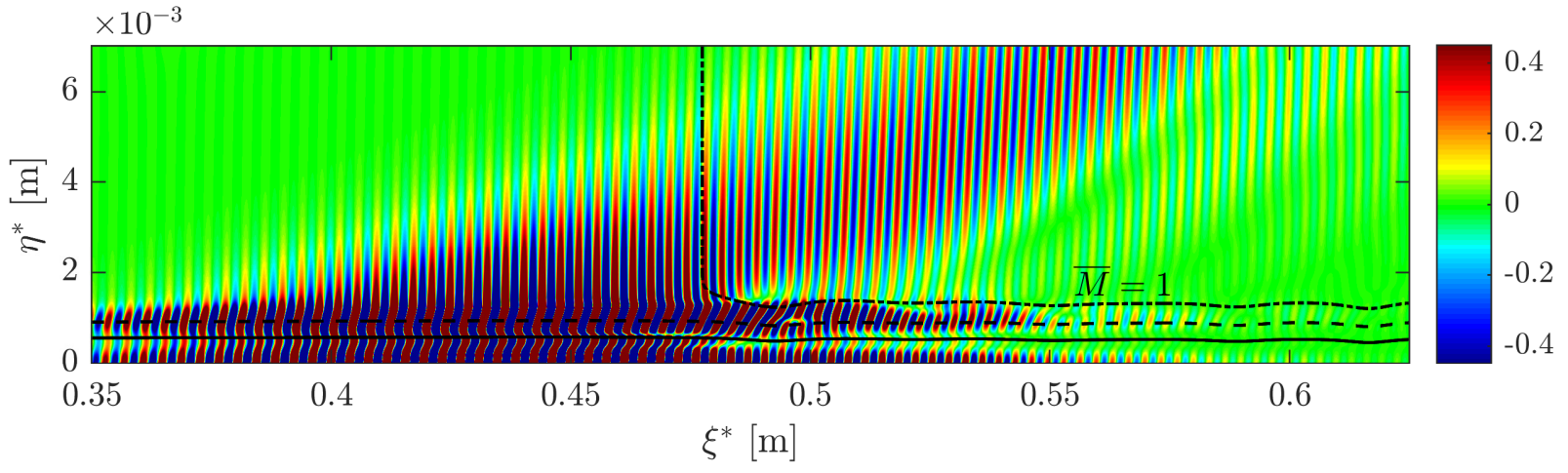

(b) Supersonic mode

Fig. 10 Specific volume perturbations from OWNS showing the second mode (top) and the supersonic mode appearing further downstream (bottom) at $R e_{\infty}=4.06 \times 10^{6} \mathrm{~m}^{-1}$ and $f=550 \mathrm{kHz}$ with Mode $S$ initialized at the inlet.

To understand the instabilities present in Figure 10, we first define the relative Mach number

$$
\bar{M}(\eta)=\frac{\bar{u}_{\xi}(\eta)-c_{r}}{\bar{a}(\eta)} .
$$

Note that we use Eq. 41 and Eq. 42 in determining $\alpha_{r}$ and $\alpha_{i}$, respectively, such that

$$
c_{r}=\operatorname{Re}\left\{\frac{\omega}{\alpha_{r}+i \alpha_{i}}\right\} .
$$

Typical of the second Mack mode, we see amplification of acoustic waves trapped between the wall and relative sonic line $\bar{M}\left(\eta_{s}\right)=-1$. In this region, disturbances travel supersonically downstream relative to the local mean flow. Outside this region, for $\bar{M}>-1$, the disturbances travel subsonically relative to the freestream and thus we observe the subsonic "rope-like" structures centered about the critical layer $\bar{M}\left(\eta_{c}\right)=0$. However, due to substantial wall-cooling $\left(T_{w} / T_{e}=0.864\right)$, there is an additional mode - the supersonic mode - which appears at $\xi^{*} \approx 0.477 \mathrm{~m}$, causing the modulation of the wall-pressure signal seen in Figure 9 , a phenomenon also observed by [24]. This mode occurs when the phase speed of the disturbances in the freestream is slow enough such that they travel supersonically upstream 
relative to the freestream $(\bar{M}>1)$ and creates an acoustic radiation from the boundary layer [19] (this was also observed for the highly-cooled flat-plate case). This acoustic emission eventually decays as Mach waves in the freestream. This validation case serves to illustrate how OWNS is once again capable of accurately modeling complex multi-modal instabilities.

Following the discussion from Section [I] one of the major disadvantages of using PSE is that it is only suited to track a dominant mode of a desired wavenumber. However, it is often desirable to perform unsteady calculations initialized with random perturbations such that the base flow dictates the unstable modes that appear. The normal-mode ansatz that PSE is based upon precludes such analyses. OWNS, however, is capable of tracking downstream-propagating modes that form from arbitrary initial conditions. We illustrate this by initializing the same stability calculation but with a normally-distributed random $\phi_{0}$ within the boundary layer and homogeneous conditions in the freestream. The wall-pressure signal from this analysis is once again compared to DNS in Figure 9 (note that there is significant overlap to the wall-pressure signal with Mode $\mathrm{S}$ initialization). After the initial transients, we observe excellent agreement to the DNS data with the appearance of the second mode, thus demonstrating the versatility of using OWNS in marching an arbitrary initial condition.

\section{HIFiRE-5 elliptic cone}

We first validate the linear operator $M$ from Eq. 12 (assuming $g=h=0$ as usual) by comparing N-factors computed using our LST code versus that of LASTRAC's [40]. Figure 11] shows the comparison at $f=60,70$, and $80 \mathrm{kHz}$ in which the $\mathrm{N}$-factor is defined as

$$
N_{L S T}=-\int_{\xi_{0}}^{\xi} \alpha_{i}\left(\xi^{\prime}\right) d \xi^{\prime}
$$

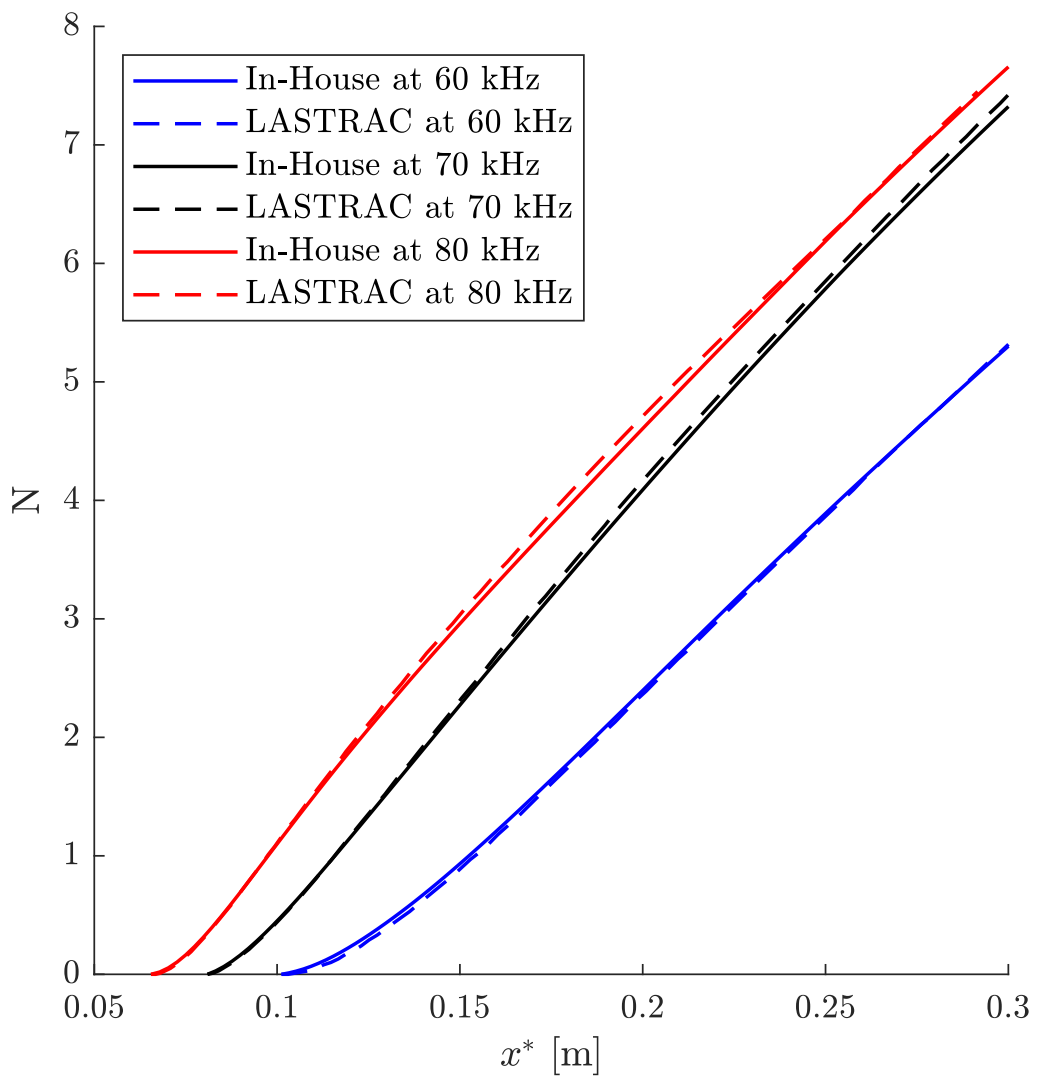

Fig. 11 N-factors along the HIFiRE-5 centerline computed with our In-House LST code versus LASTRAC for $f=60,70$, and $80 \mathrm{kHz}$.

We see excellent agreement in the $\mathrm{N}$-factors computed from our LST code and that of LASTRAC's. Thus, PSE 
and OWNS computations are performed for the same frequency range in which the second mode is used as the inlet boundary condition with $N_{b}=15$ for OWNS. We compare the N-factors from OWNS, PSE, and LST in Figure 12 where the $\mathrm{N}$-factor for OWNS and PSE is defined as

$$
N_{P S E / O W N S}=\ln \left(\frac{\left|p_{w}^{\prime}\right|}{\left|p_{w_{0}}^{\prime}\right|}\right) .
$$

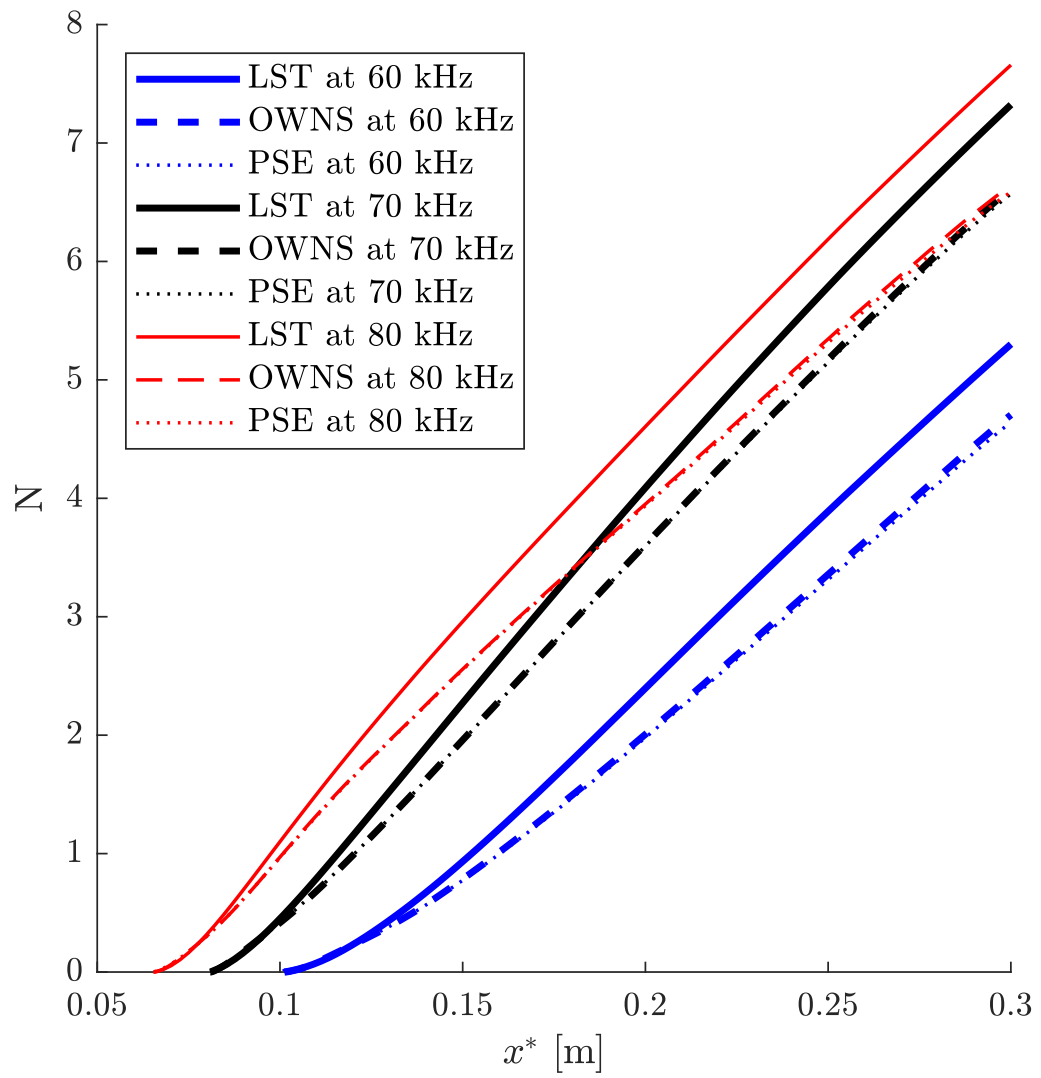

Fig. 12 Comparing the N-factor along the HIFiRE-5 centerline between LST, OWNS, and PSE for $f=60,70$, and $80 \mathrm{kHz}$.

From Figure 12, we see that for all frequencies, locally removing the parallel mean-flow assumption that is used in LST stabilizes the second mode along the centerline. This is most likely due to the unusually large $\bar{u}_{\eta}$ from the primary vortex roll-up advecting the perturbation energy upwards and away from the wall. To understand why PSE and OWNS performed almost identically, we visualize the disturbance field by plotting the specific volume perturbations at $f=80$ $\mathrm{kHz}$ in Figure 13 . 


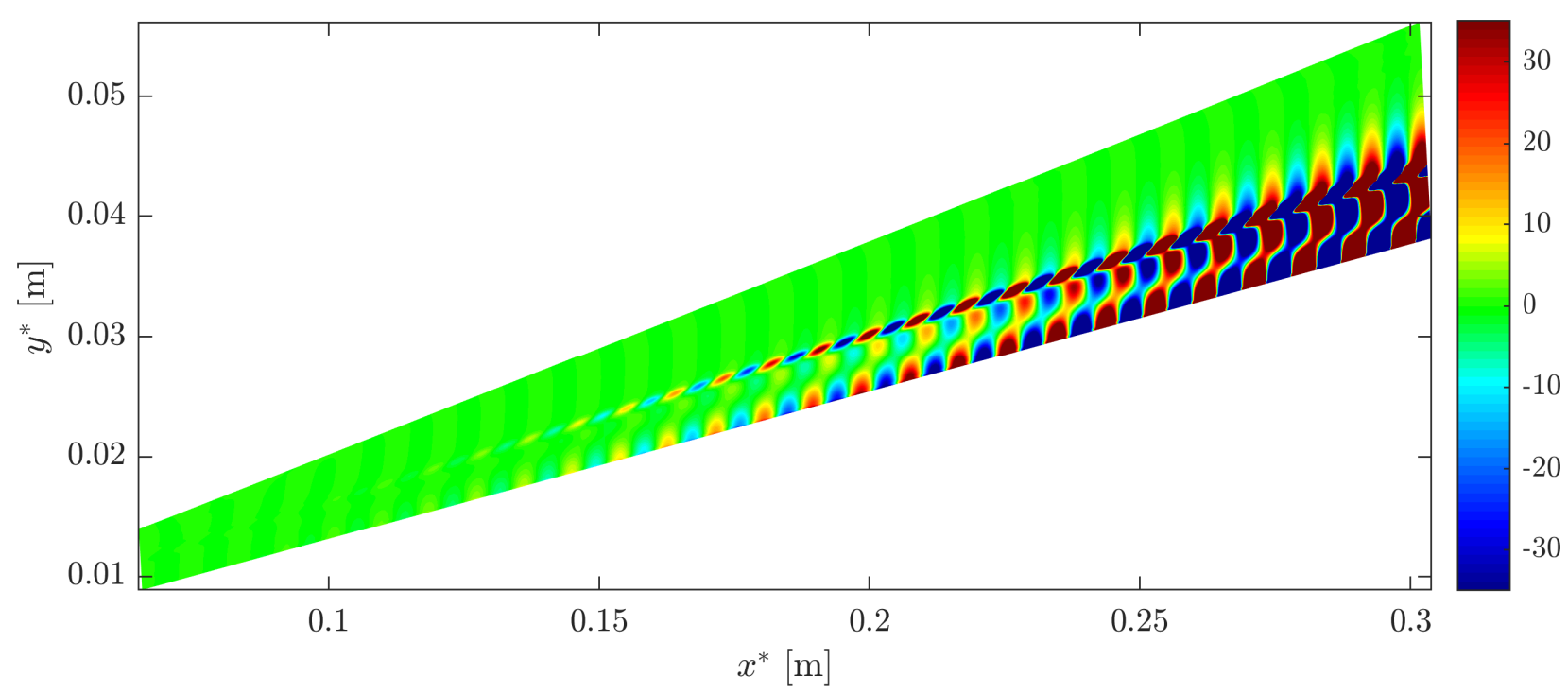

Fig. 13 Specific volume disturbance field from OWNS along the centerline of the HIFiRE-5 elliptic cone for $f=80 \mathrm{kHz}$.

Unlike validation Cases 2 and 3 where we witnessed complex multi-modal interactions, we do not observe any similar physics in Figure 13. The presence of one dominant instability is likely why PSE performed on par with OWNS. However, the peak amplitudes of the perturbations are separated by regions of high shear in the base flow (refer to Figure 4). This is contrary to typical second-mode wave structure as seen in validation Case 3 and alludes to potentially additional unstable modes not revealed under a strictly two-dimensional analysis. In fact, the presence of such complex mode shapes along a plane of symmetry may indicate that a fully three-dimensional stability calculation is required to accurately track the disturbance evolution (this is somewhat expected since the primary vortex roll-up near the leeward line induces strong spanwise variations [42]).

\section{Conclusion and Future Work}

We extended the OWNS framework to hypersonic boundary layers with full compressibility under a general, non-orthogonal curvilinear coordinate system, and validated the methodology by comparing to DNS results (in the linear regime) of an adiabatic flat plate, a highly-cooled flat plate, and a 7-deg half-angle sharp cone. In all cases, we observe that OWNS gives DNS-quality results at a tiny fraction of the computational expense. Essentially, OWNS was able to fully track the downstream-propagating modes even when initialized with random perturbations since the algorithm uses a high-order recursive filter to remove upstream disturbances. Additionally, we validated the non-orthogonal curvilinear coordinate system by comparing OWNS with LST data of the HIFiRE-5 elliptic cone computed using LASTRAC.

In future, we plan to continue to develop the OWNS framework and apply it to three-dimensional flows where the computational expense of OWNS is expected to be significantly reduced compared to global approaches. As previously demonstrated [43], OWNS is readily adapted, via an iterative method, to computing optimal resolvent (input/output) modes. Likewise, nonlinear extensions of the OWNS framework are underway.

\section{Acknowledgments}

This work has been supported by The Boeing Company through the Strategic Research and Development Relationship Agreement CT-BA-GTA-1. We also acknowledge support of the Natural Sciences and Engineering Research Council of Canada via the Postgraduate Doctoral Scholarship (PGSD3-532522-2019).

\section{References}

[1] Hirschel, E. H., Laminar-Turbulent Transition and Turbulence in High-Speed Viscous Flow, chapter and pages, pp. $263-310$. 
[2] Fedorov, A., "Transition and Stability of High-Speed Boundary Layers," Annual Review of Fluid Mechanics, Vol. 43, No. 1, 2011, p. 79-95.

[3] Zhong, X., and Wang, X., "Direct Numerical Simulation on the Receptivity, Instability, and Transition of Hypersonic Boundary Layers," Annual Review of Fluid Mechanics, Vol. 44, No. 1, 2012, p. 527-561.

[4] Mack, L. M., "Special course on stability and transition of laminar flow," AGARD report, Vol. 709, 1984.

[5] Mack, L., “Boundary-Layer Stability Theory. Part B., Jet Propulsion Lab., Pasadena, CA,” Document 900-277, Rev. A, 1969.

[6] Sivasubramanian, J., and Fasel, H. F., "Numerical investigation of the development of three-dimensional wavepackets in a sharp cone boundary layer at Mach 6," Journal of Fluid Mechanics, Vol. 756, 2014, p. 600-649.

[7] Sivasubramanian, J., and Fasel, H. F., "Direct numerical simulation of transition in a sharp cone boundary layer at Mach 6: fundamental breakdown," Journal of Fluid Mechanics, Vol. 768, 2015, p. 175-218.

[8] Sivasubramanian, J., Sandberg, R. D., Terzi, D. A. V., and Fasel, H. F., "Numerical Investigation of Transitional Supersonic Base Flows with Flow Control,” Journal of Spacecraft and Rockets, Vol. 44, No. 5, 2007, p. 1021-1028.

[9] Demetriades, A., "An experiment on the stability of hypersonic laminar boundary layers," Journal of Fluid Mechanics, Vol. 7, No. 3, 1960, p. 385-396.

[10] Demetriades, A., "Hypersonic viscous flow over a slender cone. III - Laminar instability and transition," 7 th Fluid and PlasmaDynamics Conference, 1974.

[11] Flscher, M. C., and Wagner, R. D., "Transition and Hot-wire Measurements in Hypersonic Helium Flow," AIAA Journal, Vol. 10, No. 10, 1972, p. 1326-1332.

[12] Kendall, J., "Wind Tunnel Experiments Relating to Supersonic and Hypersonic Boundary-Layer Transition," AIAA Journal, Vol. 13, No. 3, 1975, p. 290-299.

[13] Kimmel, R. L., Demetriades, A., and Donaldson, J. C., "Space-time correlation measurements in a hypersonic transitional boundary layer," AIAA Journal, Vol. 34, No. 12, 1996, p. 2484-2489.

[14] Stetson, K., and Kimmel, R., "On hypersonic boundary-layer stability,” 30th Aerospace Sciences Meeting and Exhibit, 1992.

[15] Stetson, K., Kimmel, R., Donaldson, J., and Siler, L., "A comparison of planar and conical boundary layer stability and transition at a Mach number of 8," 22nd Fluid Dynamics, Plasma Dynamics and Lasers Conference, 1991.

[16] Stetson, K., "Nosetip bluntness effects on cone frustum boundary layer transition in hypersonic flow," 16th Fluid and Plasmadynamics Conference, 1983.

[17] Fedorov, A., and Tumin, A., "High-Speed Boundary-Layer Instability: Old Terminology and a New Framework," AIAA Journal, Vol. 49, No. 8, 2011, p. 1647-1657.

[18] Saric, W. S., “Görtler Vortices,” Annual Review of Fluid Mechanics, Vol. 26, No. 1, 1994, p. 379-409.

[19] Knisely, C. P., and Zhong, X., "Sound radiation by supersonic unstable modes in hypersonic blunt cone boundary layers. II. Direct numerical simulation," Physics of Fluids, Vol. 31, No. 2, 2019, p. 024104.

[20] Arnal, D., and Casalis, G., "Laminar-turbulent transition prediction in three-dimensional flows," Progress in Aerospace Sciences, Vol. 36, No. 2, 2000, p. 173-191.

[21] Saric, W. S., Reed, H. L., and White, E. B., "Stability and Transition of Three-Dimensional Boundary Layers," Annual Review of Fluid Mechanics, Vol. 35, No. 1, 2003, p. 413-440.

[22] Kuehl, J., and Paredes, P., “Gortler Modified Mack-modes on a Hypersonic Flared Cone,” 54th AIAA Aerospace Sciences Meeting, 2016.

[23] Bitter, N. P., and Shepherd, J. E., "Stability of highly cooled hypervelocity boundary layers," Journal of Fluid Mechanics, Vol. 778, 2015, p. 586-620.

[24] Chuvakhov, P. V., and Fedorov, A. V., "Spontaneous radiation of sound by instability of a highly cooled hypersonic boundary layer," Journal of Fluid Mechanics, Vol. 805, 2016, p. 188-206. 
[25] Ma, Y., and Zhong, X., "Receptivity of a supersonic boundary layer over a flat plate. Part 1. Wave structures and interactions," Journal of Fluid Mechanics, Vol. 488, 2003, p. 31-78.

[26] Towne, A., Rigas, G., and Colonius, T., "A critical assessment of the parabolized stability equations," Theoretical and Computational Fluid Dynamics, Vol. 33, No. 3-4, 2019, p. 359-382.

[27] Towne, A., and Colonius, T., "One-way spatial integration of hyperbolic equations," Journal of Computational Physics, Vol. 300, 2015, p. 844-861.

[28] Rigas, G., Colonius, T., and Beyar, M., "Stability of wall-bounded fows using one-way spatial integration of Navier-Stokes equations," 55th AIAA Aerospace Sciences Meeting, 2017.

[29] Demirdzic, I., Gosman, A., Issa, R., and Peric, M., "A calculation procedure for turbulent flow in complex geometries," Computers \& Fluids, Vol. 15, No. 3, 1987, p. 251-273.

[30] Mattsson, K., and Nordström, J., "Summation by parts operators for finite difference approximations of second derivatives," Journal of Computational Physics, Vol. 199, No. 2, 2004, p. 503-540.

[31] Towne, A., and Colonius, T., "Improved Parabolization of the Euler Equations," 19th AIAA/CEAS Aeroacoustics Conference, 2013.

[32] Towne, A., "Advancements in jet turbulence and noise modeling: accurate one-way solutions and empirical evaluation of the nonlinear forcing of wavepackets," Ph.D. thesis, California Institute of Technology, 2016.

[33] Malik, M., "Numerical methods for hypersonic boundary layer stability," Journal of Computational Physics, Vol. 86, No. 2, 1990, p. 376-413.

[34] Sousa, V. C. B., Patel, D., Chapelier, J.-B., Wartemann, V., Wagner, A., and Scalo, C., "Numerical Investigation of Second-Mode Attenuation over Carbon/Carbon Porous Surfaces," Journal of Spacecraft and Rockets, Vol. 56, No. 2, 2019 , p. $319-332$.

[35] Wagner, A., "Passive Hypersonic Boundary Layer Transition Control Using Ultrasonically Absorptive Carbon-Carbon Ceramic with Random Microstructure," Ph.D. thesis, Katholieke Universiteit Leuven, 2014.

[36] Taylor, G. I., and Maccoll, J. W., "The air pressure on a cone moving at high speeds._-I," Proceedings of the Royal Society of London. Series A, Vol. 139, No. 838, 1933, p. 278-297.

[37] Lassaline, J. V., "Supersonic Right Circular Cone at Zero Angle of Attack," http://e.roohi .profcms .um.ac.ir/imagesm/ 1019/stories/PDFs/Supersonic/conenotes.pdf 2009. Online; Accessed: 1 May 2020.

[38] Mangler, W., "Zusammenhang zwischen ebenen und rotationssymmetrischen Grenzschichten in kompressiblen Flüssigkeiten," ZAMM - Zeitschrift für Angewandte Mathematik und Mechanik, Vol. 28, No. 4, 1948, p. 97-103.

[39] Lees, L., "Laminar Heat Transfer Over Blunt-Nosed Bodies at Hypersonic Flight Speeds," Journal of Jet Propulsion, Vol. 26, No. 4, 1956, p. 259-269.

[40] Chang, C.-L., "LASTRAC.3d: Transition Prediction in 3D Boundary Layers," 34th AIAA Fluid Dynamics Conference and Exhibit, 2004.

[41] Thompson, K. W., "Time dependent boundary conditions for hyperbolic systems," Journal of Computational Physics, Vol. 68, No. 1, 1987, p. 1-24.

[42] Li, F., Choudhari, M., Chang, C.-L., White, J., Kimmel, R., Adamczak, D., Borg, M., Stanfield, S., and Smith, M., "Stability Analysis for HIFiRE Experiments," 42nd AIAA Fluid Dynamics Conference and Exhibit, 2012.

[43] Rigas, G., Schmidt, O. T., Colonius, T., and Brès, G. A., "One Way Navier-Stokes and resolvent analysis for modeling coherent structures in a supersonic turbulent jet," 23rd AIAA/CEAS Aeroacoustics Conference, 2017. 